\title{
1 Multinucleation Associated DNA Damage causes 2 quiescence despite compromised p53
}

3

4 Madeleine Hart ${ }^{1}$, Sophie D Adams ${ }^{1}$ and Viji M Draviam ${ }^{1, \#}$

$5 \quad$ \#Correspondence to v.draviam@gmul.ac.uk

6

$7{ }^{1}$ School of Biological and Chemical Sciences, Queen Mary University of London, 8 London, UK.

Address correspondence to:

24 Viji M Draviam

25 School of Biological and Chemical Sciences

26 Queen Mary University of London

27 London E1 4NS 


\section{ABSTRACT}

30 Nuclear atypia is one of the earliest hallmarks of cancer progression. How distinct forms of

31 nuclear atypia differently impact cell fate is not understood at the molecular level. Here, we

32 perform single-cell tracking studies to determine the immediate and long-term impact of

33 multinucleation or misshapen nuclei and reveal a significant difference between

34 multinucleation and micronucleation, a catastrophic nuclear atypia known to promote

35 genomic rearrangements and tumour heterogeneity. Tracking the fate of newborn cells

36 exhibiting various nuclear atypia shows that multinucleation, unlike other forms of nuclear

37 atypia, blocks proliferation in p53-compromised cells. Because compromised p53 is seen in

38 over $50 \%$ of cancers, we explored how multinucleation blocks proliferation and promotes

39 quiescence. Multinucleation increases 53BP1-decorated nuclear bodies (DNA damage

40 repair platforms), along with a heterogeneous reduction in transcription and protein

41 accumulation across the multi-nucleated compartments. Importantly, Multinucleation

42 Associated DNA Damage (MADD) associated 53BP1-bodies remain unresolved for days,

43 despite an intact NHEJ machinery that repairs laser-induced DNA damage within minutes.

44 This persistent MADD signalling blocks the onset of DNA replication and is associated with

45 driving proliferative $\mathrm{G} 1$ cells into quiescence, revealing a novel replication stress

46 independent cell cycle arrest caused by mitotic lesions. These findings call for segregating

47 protective and prohibitive nuclear atypia to inform therapeutic approaches aimed at limiting

48 tumour heterogeneity. 


\section{INTRODUCTION}

Nuclear atypia is associated with disease states, and is a strong discriminator of survival in many cancers ${ }^{1,2}$. Micronuclei harbour nuclear envelope defects ${ }^{3}$, leading to cell

cycle asynchronicity between primary and micronucleus ${ }^{4}$ and extensive DNA damage

following replication stress ${ }^{4,5}$. Furthermore, the erroneous repair of the micronuclear $D N A^{5,6}$ promotes large scale translocations confined to micronuclear $D N A^{5}$. Thus, micronuclei can propagate genomic instability and therefore, considered tumourigenic, particularly given their ability to proliferate in p53 null conditions ${ }^{4,7}$. While micronuclei studies provide evidence that nuclear atypia may be causal to a disease state, what impact other nuclear atypia have upon a disease state is largely unknown.

Multinucleation, another form of nuclear atypia with multiple nuclear compartments, has been linked to mitotic slippage ${ }^{8}$ the erroneous exit from a prolonged mitotic arrest. Whilst multinucleated cells are known to display DNA damage ${ }^{8-10}$, their cell cycle fate compared to micronucleated cells and the dynamics of DNA damage and downstream signalling have not been assessed.

Mitotic errors can lead to a variety of nuclear atypia, including micronucleation, multinucleation or misshapen nuclei, arising either as a result of mitotic slippage (slow exit) or spindle checkpoint failure (abrupt exit) (reviewed in ${ }^{11}$ ). While structural aneuploidies can impair cell cycle progression, ${ }^{12}$ the cell cycle impact of various nuclear atypia has not been compared so far within a common mitotic lesion. A molecular understanding how nuclear atypia impacts cell viability and DNA repair status in single-cells of a population is important to understand disease outcome heterogeneity.

\section{RESULTS}

\section{Unlike other nuclear atypia, multinucleation arrests cells at G1-S, independent}

To mimic mitotic drug treatment associated nuclear atypia, we exposed cells to GSK923295, a CENP-E inhibitor (CENPEi), which disrupts the end-on conversion of chromosome-microtubule attachment ${ }^{13,14}$ leading to chromosome misalignment and a mitotic arrest ${ }^{15}$ causing mitotic slippage and a variety of nuclear atypia. To analyse cell cycle status, we immunostained for PCNA foci (S-phase marker) two days after the release from CENPEi treatment (Fig. 1A). Using immunostaining we compared 100s of RPE1 cells displaying a variety of nuclear atypia. As expected, multinucleation, micronucleation or misshapen nuclei were all observed predominantly in the CENPEi-treated but not DMSO-treated population 
displaying micronuclei or normal or misshapen nuclei presented a small proportion of PCNA foci positive cells (Fig. 1B and 1D), showing a multinucleation associated block in G1-S transition and replication initiation.

The complete absence of PCNA foci in multinucleated cells is somewhat at odds with reports of a positive association between multinucleation and tumorigenic potential and resistance to therapy ${ }^{16,17}$. So, we investigated whether the G1-S block in multinucleated cells may be lost following compromised p53 function, a tumour suppressor frequently lost in cancers (reviewed by ${ }^{18}$ ). A p53 dependent G1-S block has been reported in micronucleated cells $^{7}$, tetraploid cells ${ }^{19}$ and chromosomal missegregation or aneuploidy ${ }^{7,20,21}$, but not tested in multinucleated cells. We exposed RPE1 p53 knockdown cell line, with demonstrably no p53 expression ${ }^{7}$ (Supplementary Figure 1A, 1B) to CENP-E inhibitor and monitored PCNA status 48 hours after inhibitor wash-off (Fig. 1A). In p53 knockdown cells, where all three forms of nuclear atypia could be observed (Fig. 1F), nuclear PCNA foci, distinctly brighter than non-specific cytoplasmic speckles, were absent in multinucleated cells alone (Fig. 1E and $1 \mathrm{G})$. In contrast, cells presenting other nuclear atypia, including micronucleated or misshapen nuclei showed an increase in the proportion of PCNA foci in p53 knockdown cells compared to WT RPE1 cells (Fig. 1E and 1G). The lack of PCNA foci selectively in multinucleated cells shows that unlike other forms of nuclear atypia, multinucleation blocks G1-S transition, independent of p53 status.

Two other mitotic lesions where multinucleation is not readily induced due to accelerated mitotic exit, inhibitions of Mps1 or Aurora-B activity, yielded similar results (Supplementary Fig. 2A, 2B). Assessing the cell cycle impact of micronuclei and misshapen nuclei showed that in p53 WT conditions both treatments exhibit a G1 arrest, but less robust than multinucleate cells (Supplementary Fig. 2C). Additionally, in p53 knockdown cells, the G1 arrest was alleviated in micronucleated and misshapen nuclei caused following MPS1 or Aurora-B inhibition (Supplementary Fig. 2D, 2F). In summary, unlike other nuclear atypia, multinucleation uniquely blocks G1-S despite a compromised p53 status.

\section{Multinucleate cells exhibit the highest incidence of DNA damage}

We compared the extent of DNA damage in the various nuclear atypia, by immunostaining for gamma H2AX (double-stranded DNA damage marker), 1 and 48 hours after CENPEi washout. 48 hours after washout, $40 \%$ of micronuclei bearing cells displayed gH2AX foci exclusively within the micronuclei compartment, as reported ${ }^{4,5,22}$ and only $30 \%$ of their primary nuclei presented gH2AX foci (Supplementary Fig. 3A and 3B). In contrast, $>90 \%$ of multinucleated cells displayed $\mathrm{gH} 2 \mathrm{AX}$ foci in most of the compartments (Supplementary Fig. 3A and 3B). Importantly, the number of $\mathrm{gH} 2 \mathrm{AX}$ foci is strikingly higher after multinucleation compared to other forms of nuclear atypia (Supplementary Fig. $3 \mathrm{C}$ ), 
although the intensity of individual $\mathrm{gH} 2 \mathrm{AX}$ foci per se was not different (Supplementary Fig. 3D). Similarly, within an hour of drug washout, gH2AX foci number was strikingly higher in multinucleated cells (Supplementary Fig. 4, see 4E). These observations show that multinucleated cells are prone to a substantially higher incidence of DNA damage compared to other forms of nuclear atypia.

Next, we analysed the incidence of RIF1, an NHEJ repair pathway member that accumulates at DNA breaks specifically in $\mathrm{G} 1$ phase $\mathrm{p}^{23-25}$. Immunostaining studies showed the normal accumulation of RIF1 in the vast majority of $\mathrm{gH} 2 \mathrm{AX}$ foci in multinucleated cells, except for the gH2AX foci in micronucleated compartments (Supplementary Figure 3F). We conclude that Multinucleation associated DNA damage (MADD) successfully accumulates RIF1, a factor that promotes NHEJ in G1.

\section{Multinucleate cells exhibit delayed DNA damage signalling}

To identify the precise timing of MADD and the extent of MADD resolution, we used time-lapse microscopy to track the arrival and departure of 53BP1 foci, a DNA damage response factor downstream of gH2AX and upstream of RIF1. In RPE1 cells coexpressing 53BP1-GFP and Histone-2B-GFP (DNA marker), we compared multinucleated cells arising from a slow mitotic slippage or rapid mitotic exit following either CENP-Ei treatment alone or CENP-Ei and Aurora-Bi co-treatment, respectively (Supplementary Fig. 5A; Fig. 2A and 2B; Supplementary Videos $1 A-C)$. Videos of CENPEi washout showed nearly $80 \%$ of cells exiting mitosis with a multinucleate phenotype and only $20 \%$ of cells exhibiting either a normal or misshapen nuclei (Supplementary Fig. 5B). 53BP1 foci could be observed in multinucleated cells, as well as those with normal or misshapen nuclei (Fig. 2C), consistent with $\mathrm{gH} 2 \mathrm{AX}$ status (Supplementary Fig. 4), with multinucleated cells gaining foci in $\sim 60 \%$ of cells during the course of imaging irrespective of slow mitotic slippage (CenpEi) or a rapid mitotic exit (CenpEi \& AuroraBi). Analysis of live-cell videos revealed that the timing of 53BP1 foci arrival varies depending on the nuclear atypia: in multinucleated cells, 53BP1 foci arrival is delayed, $\sim 2-11$ hours after mitotic exit (Fig. 2D), whereas in others foci arrival was complete, within the first 4 hours after mitotic exit (Fig. 2D). In fixed-cell studies, gH2AX foci (Supplementary Fig.4) appear within 1 hour following drug washout, showing a delayed 53BP1 response in multinucleated cells.

In all multinucleated cells, irrespective of slow mitotic slippage or rapid mitotic exit, 53BP1 foci resolution was lacking and the foci number was steadily increasing in late G1 (Supplementary Fig. 5C, Fig. 2E). These single-cell tracking studies reveal that while none of the multinucleated cells resolved 53BP1 foci completely ( $n=55$ ), 40-50\% of normal nuclei displayed 53BP1 foci disappearance indicative of damage clearance $(n=8$ DMSO and 9 
159 CENPEi cells) (Supplementary Fig. 5C). We conclude that MADD signalling is delayed and

160 MADD remains unresolved.

161

162

163

\section{MADD-associated 53BP1 remains unresolved despite intact DDR in} multinucleated cells

We investigated whether MADD can not be resolved or multinucleated cells are DNA Damage Repair (DDR) deficient by tracking the fate of laser-induced DNA damage in 53BP1-GFP expressing multinucleated cells. Comparing images before and after laserinduced damage showed that cells displaying multinucleation or normal nuclei can rapidly recruit 53BP1-GFP at the laser-damage site (Fig. 3A). The acquisition of laser-induced 53BP1-GFP foci was slightly reduced in number and delayed in multinucleated cells compared to normal cells (Fig. 3B and 3C). Clearance of laser-induced 53BP1 foci was surprisingly slightly more frequent in multinucleated cells compared to normal cells (Fig. 3D). Comparing the rates of laser-induced 53BP1 foci clearance showed no difference between a multinucleated versus normal cell (Fig. 3E and 3F), revealing a proficient DDR pathway in multinucleated cells. Despite DDR proficiency, multinucleated cells did not resolve any of the non-laser induced 53BP1 foci, while normal nuclei resolved $40 \%$ of the foci during imaging of up to 15 hours (Fig. 3E). These data show that MADD associated 53BP1 foci cannot be resolved despite a proficient DDR response in multinucleated cells.

\section{Multinucleation promotes quiescence}

Tracking the impact of multinucleation on cell cycle progression, using time-lapse microscopy, revealed an important heterogeneity across multinucleate compartments. In non-transformed hTERT-RPE1 cells expressing mRuby-PCNA (S-phase Marker) and p21GFP (G1-phase marker), normal nuclei in both CENP-Ei or DMSO treated conditions showed normal appearance and disappearance of PCNA foci (CENP-Ei $=71$; DMSO =195 cells; Supplementary Videos 2A, B; Fig. 4A and 4B). In contrast, cells displaying multinucleation showed no PCNA foci formation for at least 14 hours after mitosis, despite the accumulation of nuclear mRuby-PCNA levels in most compartments (114 Cells; Sup Video 2C; Fig. 4B). Consistent with the lack of PCNA foci, multinucleated cells showed an increase in nuclear p21-GFP, 4-5 hours after mitotic exit (Fig. 4B and 4C). Increasing p21GFP signal was observed in multinucleated cells despite its absence at mitotic exit (Supplementary Video 2C, Fig. 4B), indicating a stress signalling response in late G1. Importantly, a variable increase in the rates and amount of p21 accumulation across different compartments of multinucleated cells was observed (Fig. 4C; $n=3$ cells). In summary, 
194

195

196

197

198

199

200

201

202

203

204

205

206

207

208

209

210

211

212

213

214

215

216

217

218

219

220

221

222

223

224

225

226

227

228

229

230

multinucleation promotes a steady increase in nuclear p21, heterogeneously across compartments, disallowing the onset of DNA replication.

We hypothesised that if multinucleated cells are not dead and multinucleation disrupts genome-wide function, we should see signs of altered transcription, and we were curious to see if the transcription can occur at MADD-sites. To test whether MADD heterogeneously prevents the function of transcriptional machinery, we immunostained using antibodies against gH2AX and either RNA-Pol II pSer5 (initiation marker) or RNA-Pol II pSer2 (elongation marker). Both markers were present irrespective of nuclear shapes (Fig. 4D; Supplementary Fig. 6C) showing the ability to localise transcriptional machinery and phosphorylate key sites to enable transcriptional initiation and elongation despite large-scale fragmentation of nuclear compartments in multinucleated cells. However, transcriptionassociated foci did not overlap with $\mathrm{gH} 2 \mathrm{AX}$ demonstrating a shutdown of transcription proximal to MADD sites (Fig. 4D; Supplementary Fig. 6C), similar to transcription exclusion at 53BP1 foci arising from replication stress ${ }^{26}$.

Quantifying RNA-Pol II pSer5 or pSer2 foci intensities in nuclei or within a nuclear compartment revealed a statistically significant decrease in mean intensities in some compartments of both multinucleated and micronucleated cells (Fig. 4E; Supplementary Fig. 6D). To investigate whether RNA-Pol intensities are dependent on nuclear compartment size, we correlated changes in mean intensity and compartment size. Micronucleated cells had reduced mean intensities in micronuclei but not primary nuclei, while the compartments in multinucleated cells displayed a unique heterogenous spread of intensities (Fig. 4F; Supplementary Fig. 6E). We tested whether reduced RNA-Pol intensities reflect a reduction in RNA-Pol at each transcriptional site or due to a reduction in the number of transcriptional sites. The proportion of nucleus occupied by RNA-Pol pSer5 or pSer2 foci was reduced in micronucleated and some smaller compartments of multinucleated cells (Supplementary Fig. $6 \mathrm{~A}$ and $6 \mathrm{~F})$. In smaller multinucleate compartments (<40um2) a transcriptional variability is accounted for by a variation in the number of transcriptional sites (Supplementary Fig. 6B and 6G), revealing a nuclear compartment size cut-off for transcription in multi-nucleated cells. These data show exclusion of transcription at MADD sites and a size-dependent reduction in transcription in multinucleated and micronucleated compartments. In summary, the studies of RNA-pol phosphorylation provide three key insights: (i) multinucleated cells are not dead and are transcriptionally active; (ii) multinucleation induces heterogeneity within nuclear compartments with respect to transcriptional initiation and elongation and (iii) transcriptionally active regions are clearly excluded from MADD sites, indicating the wide impact of MADD to the nearby genome.

Because multinucleated cells cannot resolve MADD (Fig. 3) and display increasing p21 (Fig. 4), we tested whether multinucleated cells would remain arrested at G1-S or enter 
231

232

233

234

235

236

237

238

239

240

241

242

243

244

245

246

247

248

249

250

251

252

253

254

255

256

257

258

259

260

261

262

263

264

265

266

267

quiescence. To address this, we allowed multinucleated cells to grow for 2 days and assessed DNA damage signalling and cell cycle markers, p53 and phospho-Rb (pRb), a proliferation marker hypophosphorylated in quiescent cells. p53 levels increase in CENPEitreated cultures as expected following a prolonged mitotic arrest and DNA damage (Supplementary Fig. 1B) and cells were predominantly negative for pRb, unlike controls (Fig. $5 A$ ).-To investigate whether entry into quiescence is varied across different forms of atypia, we performed single-cell studies, 2 days after release from mitotic arrest. The proportion of pRb-positive cells was reduced in general but significantly in multinucleated cells (Fig. 5B and $5 \mathrm{C}$ ). Under conditions inducing DNA damage, a transient reduction in p53 is sufficient to revert quiescent cells into the proliferative state ${ }^{27,28}$. We tested whether reducing p53 will allow multinucleated cells to overcome quiescence and transition into the cell cycle. While there was an increase in total pRB levels following p53 knockdown (Compare CENP-Ei associated lanes of Fig. $5 A$ and $5 D$ ), single-cell studies revealed that this increase in pRB is not prominent in multinucleated cells compared to cells displaying other nuclear atypia (Fig. $5 \mathrm{E}$ and $5 \mathrm{~F})$. We conclude that unlike other nuclear atypia, multinucleation promotes quiescence despite compromised p53 (Fig. 5G).

\section{Discussion}

We report that multinucleated cells are robustly driven to quiescence, despite compromised p53, unlike other forms of nuclear atypia, including micronuclei. This protective aspect of multinucleated cells in blocking DNA replication and S-phase is strongly linked to (i) increasing DNA damage associated 53BP1 bodies in G1-phase which remain unresolved despite an intact NHEJ pathway (ii) heterogeneous nuclear protein localisation and function across compartments (RNA transcription and protein levels), (iii) lack of overlap between DNA damage associated $\mathrm{gH} 2 \mathrm{AX}$ and transcriptional initiation or elongation foci and (iv) increasing p21 following mitotic exit, unlike quiescence signalling that normally precedes mitosis in $\mathrm{G}^{29,30}$. We propose this combination of dysfunctional sub-cellular events associated with multinucleation uniquely stabilises p53 function, presenting a protective quiescence phenotype unlike other nuclear atypia that progress through the cell cycle.

Mitotic error induced cell cycle arrest is known, yet a heterogeneity in their cell fates remains unexplained ${ }^{31-33}$; resolving this is important for disease stratification and targeted treatment. To address this gap, we explore heterogeneity in proliferative fate following the same mitotic perturbance. Previously, classes of nuclear atypia have not been quantitatively compared, other than studies of micronuclei alone ${ }^{3-5}$. Prolonged mitosis ${ }^{34-37}$ and chromosome missegregation ${ }^{20,21,38}$ are well-established causes of p53-mediated G1-arrest. Beyond these, we reveal nuclear compartment status as a predictor of proliferative fate following mitotic error. We demonstrate that multinucleation is associated with a 
268 heterogeneous change in transcription and protein accumulation across nuclear

269 compartments, and unresolved MADD associated 53BP1 bodies in G1. Surprisingly, MADD

270 sensing, NHEJ signalling, laser-induced DDR and G1-S inhibition operate normally in

271 multinucleated cells. Unresolved 53BP1 bodies associated with MADD may be similar to

272 DSB-SCARS ${ }^{39}$ except that we observe RIF1 accumulation and $\mathrm{gH} 2 \mathrm{AX}$ foci and dynamic

273 changes to some (but not all) of the 53BP1 foci in multinucleated cells. The precise

274 molecular reason for differences in 53BP1 foci resolution is unclear and would require future

275 studies downstream of gH2AX-53BP1-RIF1. Nevertheless, the impact of multinucleation on

276 some but not all subcellular processes presents an advantage for targeted treatment of

277 cancers with multinucleated cells.

278 Micronuclei with cytoplasmic DNA mount a protective immune response $e^{40,41}$, but

279 allow proliferation despite DNA damage, in the face of compromised p53. However,

280 multinucleation blocks G1-S despite compromised p53 and presents exposed DNA, offering

281 double-protection. These may be relevant to nuclear atypia induced by cytoskeletal tension

282 (reviewed $\mathrm{in}^{42}$ ), cell-substrate stiffness ${ }^{43}$ or nuclear envelope defects (reviewed $\mathrm{in}^{44}$ ). We

283 propose that unlike other forms of nuclear atypia that show unrestrained proliferation and

284 associated further accumulation of DNA instability, multinucleation presents a protective

285 form which should be considered during pathology assessment and post-treatment with anti286 mitotics. 
FIGURES

290 Figure 1 - Unlike other nuclear atypia, multinucleation arrests cells at G1-S,

291 independent of p53. A - Experimental regime; RPE1 p53 wild type (WT) or RPE1 p53 knockdown (k.d.) were treated with DMSO or CENPE inhibitor for 16 hours, then washed out. 48 hours later cells were assessed for PCNA and nuclear status. B \& E - Images of RPE1 p53 WT (B) or p53 k.d (E) cells treated as in A. Scale 15 $\mu \mathrm{m}$, insets $5 \mu \mathrm{m}$. +/- refers to PCNA foci positive or negative nuclei displayed. Note in B a micronuclei bearing cell displaying PCNA-foci has been chosen, although the majority lack PCNA-foci. C \& F- Quantification of nuclear morphology changes after treatment of RPE1 p53 WT (C) or p53 k.d (F) cells with DMSO or CENPE inhibitor, as in A. Nuclei were classified as either Mis-shapen (MS), Micronucleated $(\mu N)$ or Multinucleated (MN). N=600 cells, 3 independent experimental repeats shown as shades of grey. Statistical analysis was using a two-way ANOVA with multiple comparisons and a confidence interval of 95\%. D \& G - Quantification of the percentage of PCNA-foci positive cells, within each nuclear morphology bin, following DMSO or CENPE inhibitor treatment, of RPE1 p53 WT (C) or p53 k.d (F) cells. N>150, 3 independent experimental repeats shown as shades of grey. Statistical analysis was using multiple unpaired t-tests, comparing each morphology after CENPE inhibition to normal nuclei after DMSO treatment.

Fig. 2 - Multinucleate cells exhibit delayed DNA damage signalling and an increase in damage foci through time. A - Representative time-lapse images of normal shaped nuclei exiting mitosis after DMSO treatment, or multinucleated cells exiting mitosis after CENPE i or CENPEi and Aurora Bi treatment as in Sup Fig. 5A. Scale $25 \mu \mathrm{m}$. Yellow arrows indicate 53BP1-GFP foci within multinucleate cells. In the 53BP1-GFP lane, GFP intensities are inverted to highlight 53BP1-foci as soon as they form (associated supplementary movies present non-inverted GFP intensities).

316 B - Graph shows the timing of mitotic exit, based on nuclear morphology of daughter cells (indicated by colour). Statistical significance was assessed using an unpaired student's t-test. ${ }^{* * *}$ indicates $p<0.0001 . \mathrm{C}-$ Graph of the proportion of daughter nuclei which gain 53BP1 foci during time-lapse imaging. $\mathrm{N}$ values below bars indicate the number of nuclei from at least three independent experimental repeats. 
322 interval. ${ }^{* *}$ indicates $p<0.001 . \mathrm{D}-$ Graph of the timing of 53BP1 foci arrival in nuclei

323 which gain 53BP1 foci, after drug treatments as indicated. Time-lapse movies as in A

324 were used to determine the earliest time-point of visible 53BP1 foci following mitotic

325 exit. Each value represents one nucleus. The colour of plotted values represents

326 nuclear morphology. E - Quantification of 53BP1 foci number per nucleus over time

327 from mitotic exit.

328

Figure 3 - MADD is not resolved despite intact DDR signalling in multinucleate

cells. RPE1 H2B-RFP 53BP1-GFP cells were treated with CENPEi for 24 hours,

washed and imaging initiated. One laser induced bleach/damage site in each nucleus was tracked. A - Representative pre-bleach, bleach (at 00:00) and post-

bleach images of nuclei - either normal nuclei without pre-existing 53BP1 foci, normal nuclei with pre-existing 53BP1 foci or multinucleate nuclei with 53BP1 foci. Yellow arrows indicate sites of bleaching and are highlighted in crops. Scale $15 \mu \mathrm{m}$; insets $5 \mu \mathrm{m}$. B - Graph shows the proportion of nuclei that gain 53BP1-GFP foci at the site of laser-induced damage, for multinucleate, normal with pre-existing foci and normal nuclei without pre-existing foci. $\mathrm{N}$ indicates the number of cells, from across 3 independent repeats. Statistical significance was assessed using a proportions test with a 95\% confidence interval. ${ }^{* *}$ indicated $p<0.05$. C - Graph shows timing, postbleach, of 53BP1-GFP foci arrival at the laser bleach site. D - Quantification of the proportion of nuclei that cleared laser-induced 53BP1-GFP foci, for normal and multinucleate cells. Statistics was assessed using a proportions test with a $95 \%$ confidence interval. ** indicates $p<0.05$. E - Graph shows the timing of 53BP1-GFP foci clearance after bleach time, for foci induced at the bleach site (solid lines) and foci existing prior to laser bleach (dashed lines). $\mathrm{N}$ indicates the number of foci and cells analysed. F - Graph shows changes in 53BP1-GFP foci intensity at laserinduced damage site in cells shown in A. 53BP1-GFP intensities were normalised using pre-laser damage intensity values).

Figure 4 - Multinucleation favours heterogeneous nuclear protein levels. $A$ \& $B$ - Representative images of RPE1 mRuby-PCNA p21-GFP cells treated with DMSO (A) or CENPEi (B) for 16 hours and washed 10 hours prior to live-cell imaging for 14 hours. Scale bar $25 \mu \mathrm{m}$. Yellow $+/$ - refers to p21 or PCNA foci positive or negative 
356

357

358

359

360

361

362

363

364

365

366

367

368

369

370

371

372

373

374

375

376

377

378

379

380

381

382

383

384

385

386

387

388

389

respectively. Images show a normal shaped nucleus with low p21 and nuclear PCNA foci and a multinucleate cell, exiting mitosis and building p21-GFP levels through time, without gaining nuclear PCNA foci. C - Graph of the mean p21 level per nucleus for the normal, or per compartment for multinucleated cells from movies as in A \& B. Values are normalised to the mean p21 value from the first time point measured. PCNA signal was used to identify nuclear areas. Colours of lines correspond to different nuclear compartments. D - RPE1 cells treated with DMSO or CENPEi for 16 hours were fixed 48 hours later for immunostaining with antibodies against gamma H2AX and RNA pol II CTD pSer5. DNA was stained with DAPI. Representative images of non-overlap between gamma H2AX and RNA pol II pSer5 in cells following DMSO or CENPEi treatment. Scale $15 \mu \mathrm{m}$ insets $5 \mu \mathrm{m}$. E - Graph of the mean RNA pol II CTD pSer5 intensity per nucleus (normal or misshapen nuclei) or nuclear compartment (micronucleated and multinucleate). Each plot represents one nucleus/nuclear compartment respectively. $\mathrm{N}$ refers to the number of cells analysed, across 3 independent repeats. Statistical significance was assessed using a one way ANOVA with multiple comparisons. F - Mean RNA pol II CTD pSer5 intensity per nucleus/nuclear compartments plotted against the size of the nucleus/nuclear compartment. Line colours represent nuclear morphology as indicated. $\mathrm{N}$ refers to the number of cells from 3 independent repeats.

Figure 5 - Multinucleation promotes quiescence. RPE1 p53 WT (A-C) or RPE1 H2B-GFP p53 k.d. (D-F) cells were treated with DMSO or CENPEi for 16 hours and 48 hours later cells were immunostained with antibodies against $\mathrm{pRb}$ and gamma H2AX, or cells were lysed for immunoblot. A \& D - Immunoblot shows pRb or gamma-tubulin levels in RPE1 p53 WT (A) or p53 kd (D) cells following DMSO or CENPEi treatment, as indicated. Note the gamma-tubulin is the same as displayed in Sup Fig 1. Right panel shows a graph of $p R b$ fluorescent intensity, normalised to gamma-tubulin. B \& E - Representative images of nuclear atypia following CENPEi treatment of RPE1 p53 WT (B) or p53 kd (E)cells. Scale 15 4 m. C \& F- Graph of proportion of $\mathrm{pRb}$ positive or negative RPE1 WT (C) or p53 kd (F) cells, after DMSO or CENPEi treatment. N>100 for WT or >150 for p53 k.d cells from 2 or 3 independent repeats (shown as shades of grey), respectively. Statistical analysis using multiple unpaired t-tests, comparing each morphology after CENPEi treatment to normal nuclei after DMSO. G - Model comparing nuclear atypia shows large-scale 
390 DNA damage in multinucleate cells, but not in misshapen nuclei, and in

391 micronucleated cells, gamma H2AX foci are majority confined to the micronucleus.

392 Nuclear atypia causes G0 arrest in p53 WT. In p53 k.d. conditions, only

393 multinucleate cells are G0 arrested.

394

Supplementary Figures

396

397

Supplementary Figure 1 - Immunoblot evidence of p53 knockdown. RPE1 p53

398

WT and RPE1 H2B-GFP p53 k.d. cells were treated with either DMSO (solvent

399

control) or CENPE inhibitor for 16 hours. Drug treatments were then washed out and

400

cells were lysed 2 days later. A - Lysates were run of SDS-PAGE gel and

401

immunoblotted with antibodies against p53 and gamma-tubulin. B - Quantification of

402

immunoblot bands; p53 band intensities were normalised to the intensity of gamma-

403

tubulin bands.

404

405

Supplementary Figure 2 - Mis-shapen and micronucleated cells have similar

406 cell cycle responses following an accelerated mitotic progression. RPE1 p53

WT or RPE1 H2B-GFP p53 k.d. cells were treated with MPS1 inhibitor or Aurora B inhibitor for 16 hours then washed out. 48 hours later cells were fixed and immunostained with an antibody against PCNA and DNA was stained with DAPI. A Representative images of RPE1 p53 WT cells treated with MPS1 or Aurora B inhibitor. Scale bar $15 \mu \mathrm{m}$, insets $5 \mu \mathrm{m}$. B - Quantification of nuclear morphology changes following indicated treatments in RPE1 p53 WT cells. N=600 cells from across 3 independent repeats. Statistical analysis was carried out using a two-way ANOVA with multiple comparisons and a confidence interval of $95 \%$. CQuantification of the percentage of PCNA-foci positive cells, within each nuclear morphology bin, following MPS1 or Aurora B inhibition. $\mathrm{N}=$ at least 150 nuclei from across 3 independent repeats shown as shades of grey. Statistical analysis was carried out using multiple unpaired t-tests, comparing each morphology after MPS1 or Aurora B inhibition to normal nuclei after DMSO treatment. D - Representative images of RPE1 H2B-GFP p53 k.d. cells treated with MSP1 or Aurora B inhibitor. 
422 following MSP1 or Aurora B inhibitor treatment in RPE1 H2B-GFP p53 k.d. cells.

$423 \mathrm{~N}=900$ cells from across 3 independent repeats. Statistical analysis was carried out

424 using a two-way ANOVA with multiple comparisons and a confidence interval of

425 95\%. F - Quantification of the percentage of PCNA-foci positive cells, within each

426 nuclear morphology bin, following MPS1 or Aurora B inhibition. after MPS1 or

427 Aurora B inhibition. $\mathrm{N}=$ at least 150 nuclei from across 3 independent repeats shown

428 as shades of grey. Statistical analysis was carried out using multiple unpaired t-tests,

429 comparing each morphology after MPS1 or Aurora B inhibition to normal nuclei after

430 DMSO treatment.

431

432

Supplementary Figure 3 - Multinucleation induces DNA damage, increased

433 nuclei with gamma H2AX foci and increased foci within each nucleus. RPE1

434

cells were treated with DMSO or CENPE inhibitor for 16 hours, then washed out and fixed 2 days later. Cells were immunostained with antibodies against $\mathrm{YH} 2 \mathrm{AX}$ and

DNA was stained with DAPI. A - Representative images of each nuclear morphology after CENPE inhibition or DMSO treatment. Images are maximum z projections of at least 4 images spanning $2 \mu \mathrm{m}$. Scale bar $15 \mu \mathrm{m}$. Yellow arrow indicates a micronucleus. B - Graph to show the percentage of each nuclear morphology which had $\mathrm{yH} 2 \mathrm{AX}$ foci within the nucleus, following DMSO or CENPE inhibition. $\mathrm{N}=300$ nuclei per morphology from across 3 independent repeats shown as shades of grey. Significance was assessed with multiple unpaired t-tests to compare each nuclear morphology after CENPE inhibition to normal nuclei after DMSO treatment. C Quantification of the number of $\mathrm{yH} 2 \mathrm{AX}$ foci per nucleus, in nuclei with $\mathrm{yH} 2 \mathrm{AX}$ foci. Quantification is divided based upon nuclear morphology after DMSO or CENPE inhibition. $\mathrm{N}$ indicated the number of cells, from across 3 independent repeats.

447 Significance was assessed using an unpaired t-test. Other nuclear morphologies 448 were non-significant when compared to normal nuclei after DMSO treatment. D 449 Quantification of the $\mathrm{yH} 2 \mathrm{AX}$ fluorescence intensity per foci, in nuclear morphologies indicated after DMSO or CENPE inhibitor treatments. Boxes represent second and third quartiles respectively, middle line displays median, and whiskers span minimum to maximum values. $\mathrm{N}$ indicated the number of cells analysed, taken across 3 independent repeats. F - RIF1 localises to sites of DNA damage in multinucleate cells, but not inside micronuclei. RPE1 cells were treated with DMSO or CENPE inhibitor for 16 hours then washed out and 2 days later fixed and immunostained with 
456 antibodies against gamma H2AX and RIF1. DNA was stained with DAPI. As a

457 positive control RPE1 cells were treated with Aphidicolin for the duration of the

458 experiment. Representative images of cells with differing nuclear morphology after

459 indicated drug treatments; Normal after DMSO ( $N=29$ cells), Normal $(N=8)$,

460 Multinucleate $(\mathrm{N}=12)$, Micronuclei $(\mathrm{N}=8)$ and Mis-shapen nuclei $(\mathrm{N}=7)$ after CENPE

461 inhibition, and normal nuclei after Aphidicolin treatment $(\mathrm{N}=8)$. Blue arrow indicates

462 gamma H2AX foci but no RIF1 localisation within micronucleus, and yellow arrow

463 indicates site of gamma H2AX and RIF1 co-localisation. Scale bar $15 \mu \mathrm{m}$.

Supplementary Figure 4 - Multinucleate cells have DNA damage 1-hour post-

drug washout. A - Experimental regime; RPE1 cells were treated with CENPE inhibitor or DMSO for 16 hours, the drug then washed out and cells were fixed 1 hour later. Cells were immunostained with antibodies against $\mathrm{yH} 2 \mathrm{AX}$ and DNA was stained with DAPI. B - Representative images of nuclei as treated as indicated in A. Scale bar $15 \mu \mathrm{m}$. Yellow arrow indicates a micronucleus. C - Quantification of the proportion of cells with abnormal nuclei after treatment as described in $A . N=900$ cells per condition, taken from 3 independent repeats. D - Quantification of the proportion of each nuclear abnormality with $\mathrm{yH} 2 \mathrm{AX}$ foci within the nucleus. $\mathrm{N}$ is indicated below bars, taken from 3 independent repeats. Statistical analysis was carried out using multiple unpaired t-tests, comparing each morphology after CENPE inhibition to normal nuclei after DMSO treatment. F - Quantification of the number of $\mathrm{YH} 2 \mathrm{AX}$ foci per nucleus, for each nuclear morphology, after DMSO or CENPE inhibitor treatment. $\mathrm{N}$ is indicated under each bar, taken from 3 independent repeats. Significance was assessed using an unpaired t-test. Other nuclear morphologies were non-significant when compared to normal nuclei after DMSO treatment. G Quantification of the fluorescence intensity of $\mathrm{yH} 2 \mathrm{AX}$, per foci, in nuclear morphologies indicated after DMSO or CENPE inhibitor treatment. Boxes represent second and third quartiles respectively, middle line displays median and whiskers span minimum to maximum values. $\mathrm{N}$ indicated the number of cells analysed, taken across 3 independent repeats. 
488 CENPE inhibitor for 24 hours, then washed out and one well additionally treated with

489 Aurora B inhibitor. Cells were then imaged for at least 12 hours with an image every

49030 minutes. B - Quantification of the nuclear morphology of daughter cells exiting

491 mitosis after DMSO, CENPE inhibitor or CENPE inhibitor plus Aurora B inhibitor

492 treatment. N, as indicated, is the number of daughter nuclei. C - Quantification of the

493 proportion of nuclei which display clearance of 53BP1 foci during time-lapse imaging.

$494 \mathrm{~N}$ values below bars indicate the number of nuclei. Statistical significance was

495 assessed using a proportions test, with a 95\% confidence interval. ${ }^{* * *}$ indicates

$496 p<0.001,{ }^{*}$ indicates $p<0.05$. D - Representative tracks of individual cells with the

497 number of 53BP1 foci over time following mitotic exit. The number and colour

498 represent the number of 53BP1 foci per nucleus.

Supplementary Figure 6 - Transcription initiation and elongation variable in smaller compartments of multinucleate cells. A - Quantification of the proportion

of the nucleus occupied by RNA pol II CTD pSer5, for each nucleus (normal and indicate the number of cells analysed. Statistical significance was assessed using a one way ANOVA with multiple comparisons. B - Plot of the proportion of the nucleus occupied by RNA pol II CTD pSer5 against nucleus or nuclear compartment size.

506 Colours of plots represent nuclear morphology as indicated. N values indicate the 507 number of cells. C-G - RPE1 cells were treated with DMSO or CENPE inhibitor for 50816 hours, then washed out and fixed 2 days later. Cells were immunostained with antibodies against $\mathrm{YH} 2 \mathrm{AX}$ and RNA polymerase II CTD pSer2 and DNA was stained with DAPI. C - Representative images of differing nuclear morphologies after

511 CENPE inhibitor treatment, and normal nuclei after DMSO treatment. Scale bar

$51215 \mu \mathrm{m}$, insets $5 \mu \mathrm{m}$. D - Quantification of the average RNA pol II CTD pSer2 intensity 513 per nucleus (normal or misshapen nuclei) or nuclear compartment (micronucleated

514 and multinucleate). Each plot represents one nucleus/nuclear compartment

515 respectively. $\mathrm{N}$ indicated below the bar is the number of cells analysed, from across

5163 independent repeats. Statistical significance was assessed using a one way

517 ANOVA with multiple comparisons. E - Average RNA pol II CTD pSer2 intensity per

518 nucleus/nuclear compartments plotted against the size of the nucleus/nuclear

519 compartment. Colours of plots represent nuclear morphology as indicated. N, as

520 indicated, is number of cells taken from across 3 independent repeats. F - 
521 Quantification of the proportion of the nucleus occupied by RNA pol II CTD pSer2,

522 for each nucleus (normal and misshapen nuclei) or nuclear compartment

523 (micronuclei and multinucleate). $\mathrm{N}$ values indicate the number of cells analysed.

524 Statistical significance was assessed using a one way ANOVA with multiple

525 comparisons. G - Graph of the proportion of the nucleus occupied by RNA pol II

526 CTD pSer5 against nucleus or nuclear compartment size. Colours of plots represent

527 nuclear morphology as indicated.

528

\section{Supplementary Videos}

530 Supplementary Videos 1 - Multinucleate cells gain 53BP1-GFP foci in G1. RPE1 H2B-

531 RFP 53BP1-GFP cells were treated with DMSO or CENPE for 24 hours. Cells were then

532 washed and one well was additionally treated with Aurora B inhibitor. Cells were then

533 imaged for at least 12 hours using time-lapse microscopy. Imaging shows 53BP1-GFP (top

534 left), H2B-RFP (top right), widefield (lower left) and merge (lower right) channels. A - Normal

535 daughter nuclei exiting mitosis following DMSO treatment, do not gain 53BP1-GFP foci

536 during the course of the imaging. B - A CENPE inhibitor arrested cell exits mitosis into a

537 multinucleate daughter cell which gains initial 53BP1-GFP foci at 08:30hrs and increases in

538 number until the end of the Video. Corresponding images in Figure 5 (first two panels)

539 present intensity adjustments to rule out any low intensity foci in early G1. C - CENPE

540 inhibitor arrested mitotic cell is released from mitosis with Aurora B inhibition and forms a

541 multinucleate daughter cell. Numerous 53BP1-GFP foci appear at 09:00hrs which increase

542 in number until the end of imaging.

543

544 Supplementary Videos 2 - Nuclear PCNA foci in normal nuclei after DMSO or CENPE

545 inhibition but not in multinucleate cells, which have increasing p21 levels. RPE1

546 mRuby-PCNA p21-GFP cells were treated with DMSO $(A)$ or CENPE inhibitor $(B, C)$ for 16

547 hours. Drugs were then washed out and live-cell imaging started 10 hours later. Imaging

548 shows p21-GFP (top left), mRuby-PCNA (top right), widefield (lower left) and merge (lower

549 right) channels. A - Normal nucleus after DMSO treatment shows nuclear PCNA foci and

550 lack of p21. B - Normal nucleus after CENPE inhibition shows nuclear PCNA foci and lack of

551 p21. C- Multinucleate cell after CENPE inhibition exits mitosis and builds p21 level through

552 time, without gaining nuclear PCNA foci. Scale bar $25 \mu \mathrm{m}$. Videos correspond to images

553 shown in Figure 2.

554 


\section{Materials and Methods}

\section{Cell culture and cell lines}

557 RPE1 cells were cultured in DMEM F12 supplemented with $10 \%$ fetal calf serum and

558 penicillin and streptomycin. For experiments they were plated onto $13 \mathrm{~mm}$ round coverslips

559 for immunofluorescence or onto glass-bottomed dishes (LabTech) for live-cell imaging. All

560 live-cell studies were conducted in stable cell lines: RPE1 H2B-RFP 53BP1-GFP, RPE1

561 H2B-GFP p53 k.d, mRuby-PCNA and p21-GFP cell lines were all grown in DMEM-F12 and

562 filmed in L15 media.

\section{Drug treatments}

564 To disrupt mitosis cells were treated with 10nM GSK923295 to inhibit CENPE, 1UM

565 Reversine to inhibit Mps1 and 10uM ZM447439 to inhibit Aurora B. For immunofluorescence

566 experiments drug treatments were incubated for 16 hours then washed off and fixed 48

567 hours later, unless indicated otherwise in text.

\section{$568 \quad$ Live-cell time-lapse imaging}

569 To image cell cycle progression in RPE1 p21-GFP mRuby-PCNA cells, cells were treated 570 with 10nM GSK923295 or DMSO control for 16 hours. Cells were then washed and then 571 additionally treated with DMSO or 10uM ZM447439. 10 hours later cells were washed and

572 media changed to Leibovitz (L15) imaging media at $37^{\circ} \mathrm{C}$ and imaging started. An image

573 was taken at least every 30 minutes for 14 hours. Images were acquired using a 40x 0.75NA

574 objective on a DeltaVision Core ${ }^{\mathrm{TM}}$ microscope (GE Healthcare) with a Cascade2 camera 575 under EM mode.

576 To image 53BP1-GFP foci arrival in cells following mitotic disruption RPE1 H2B-RFP 53BP1-

577 GFP cells were treated with DMSO or GSK923295 for 24 hours. Cells were washed and

578 then transferred to $37^{\circ} \mathrm{C}$ Leibovitz imaging media with DMSO or ZM447429 in the media.

579 Cells were then imaged for at least 12 hours with an image taken at least every 30 minutes.

580 Images were acquired using a 40x 0.75NA objective on a DeltaVision Core ${ }^{\mathrm{TM}}$ microscope

581 (GE Healthcare) with a Cascade2 camera under EM mode.

\section{FRAP photobleaching to induce 53BP1-GFP foci}

583 The Deltavision Core ${ }^{\mathrm{TM}}$ microscope was used with the FRAP tool using Quantifiable laser 584 module components (488nm laser). Target points were identified and then bleached with a 585 pulse duration of 1 second and a laser power of $30 \% .3$ pre-bleach and 3 post-bleach 
586 images were taken at each target site, all 0.5 seconds apart, using a 60x 1.42 NA objective,

587 using CoolSnap HQ camera (Photometrics). Following each site being bleached, time-lapse

588 microscope was initiated for the following 13 hours with an image acquired every 30

589 minutes, using a 40x 0.75 NA objective with a Cascade2 camera under EM mode.

\section{Immunofluorescence}

591 For immunofluorescence the following antibodies were used; PCNA (CST, 3586S, 1:1000),

592 Phospho-Rb (Ser 807/811) (CST, 8516S, 1:1000), yH2AX (Abcam, ab26350, 1:800), yH2AX

593 (CST. 1:1000), GFP (Abcam, ab290, 1:1000), RNA polymerase II CTD repeat phospho-Ser2

594 (Abcam, ab126353, 1:1000) and RNA polymerase II CTD repeat phospho-Ser5 (Abcam,

595 ab5408, 1:1000). DNA was stained with DAPI. Cells were fixed with ice-cold methanol and

596 blocked with 1\% BSA in PBS before immunostaining. Images of immunostained cells were

597 acquired using a 100x 1.2 NA objective on a DeltaVision Core microscope with a CoolSnap

$598 \mathrm{HQ}$ camera (Photometrics). Cells were scored as positive for PCNA foci when foci signal

599 intensities associated with the PCNA foci (inside the nuclei) were well above the background

600 speckles outside the nuclei.

\section{$601 \quad$ Nuclear atypia scoring}

602 DAPI stained nuclei were categorised based upon nuclear appearance, for nuclear

603 morphology. As in Figure 1B, normal nuclei were those that had a continuous nuclear

604 periphery and a regular oval shape, abnormal shaped nuclei were those that deviated from

605 this regular oval shape but the nuclear material was contained within one nuclear

606 compartment. Micronucleated cells have one or a few nuclear compartments external to the

607 primary nucleus, containing one or a fragment of a chromosome. Multinucleate cells have

608 multiple nuclear compartments varying in size, and can include micronuclei.

\section{Image analysis}

610 Image $\mathrm{J}$ was used for measurements of fluorescent intensities, using 8-bit images. Softworx 611 was used for manual image analysis of 53BP1-GFP foci tracking.

\section{Statistical analysis}

613 Graphs were plotted using Graphpad Prism, which was also used for statistical testing,

614 except form proportions tests which were carried out in Microsoft Excel. In all graphs

615 presented error bars represent standard deviation. In statistical tests presented the following

616 indications for $p$ values were used; non-significant $-n s$ for $p>0.05$, * for $p<0.05$, ${ }^{* *}$ for

$617 p<0.01,{ }^{* * *}$ for $p><0.001,{ }^{* * * *}$ for $p><0.0001$. 


\section{Acknowledgements}

619 The work was supported by funding to Draviam by a BBSRC research grant (R01003X/1)

620 and QMUL start-up grant (SBC8DRA2). We thank the Medema and Barr labs for providing

621 RPE1 H2B-RFP 53BP1-GFP cell line and RPE1 H2B-GFP p53 k.d. cell line as gifts.

622

\section{REFERENCES}

624 1. Kadota, K. et al. A nuclear grading system is a strong predictor of survival in epitheloid 625 diffuse malignant pleural mesothelioma. Mod. Pathol. 25, 260-271 (2012).

626 2. Kadota, K. et al. A grading system combining architectural features and mitotic count

627 predicts recurrence in stage I lung adenocarcinoma. Mod. Pathol. 25, 1117-1127 $628 \quad$ (2012).

629 3. Hatch, E. M., Fischer, A. H., Deerinck, T. J. \& Hetzer, M. W. Catastrophic nuclear 630 envelope collapse in cancer cell micronuclei. Cell 154, 47-60 (2013).

631 4. Crasta, K. et al. DNA breaks and chromosome pulverization from errors in mitosis.

$632 \quad$ Nature 482, 53-58 (2012).

633 5. Zhang, C.-Z. et al. Chromothripsis from DNA damage in micronuclei. Nature 522, 179$634184(2015)$.

635 6. Ly, P. et al. Selective $Y$ centromere inactivation triggers chromosome shattering in 636 micronuclei and repair by non-homologous end joining. Nat. Cell Biol. 19, 68-75 (2017).

637 7. Soto, M. et al. p53 Prohibits Propagation of Chromosome Segregation Errors that

638 Produce Structural Aneuploidies. Cell Rep. 19, 2423-2431 (2017).

639 8. Brito, D. A. \& Rieder, C. L. Mitotic checkpoint slippage in humans occurs via cyclin B 640 destruction in the presence of an active checkpoint. Curr. Biol. 16, 1194-1200 (2006).

641 9. Zhu, Y., Zhou, Y. \& Shi, J. Post-slippage multinucleation renders cytotoxic variation in 642 anti-mitotic drugs that target the microtubules or mitotic spindle. Cell Cycle 13, 1756$643 \quad 1764(2014)$.

644 10. S. Pedersen, R. et al. Profiling DNA damage response following mitotic perturbations. 
$645 \quad$ Nat. Commun. 7, 13887 (2016).

646 11. Shi, J. \& Mitchison, T. J. Cell death response to anti-mitotic drug treatment in cell

647 culture, mouse tumor model and the clinic. Endocr. Relat. Cancer 24, T83-T96 (2017).

648 12. Soto, M., García-Santisteban, I., Krenning, L., Medema, R. H. \& Raaijmakers, J. A.

649 Chromosomes trapped in micronuclei are liable to segregation errors. J. Cell Sci. 131,

$650 \quad$ (2018).

651 13. Shrestha, R. L. \& Draviam, V. M. Lateral to end-on conversion of chromosome-

652 microtubule attachment requires kinesins CENP-E and MCAK. Curr. Biol. 23, 1514-

$653 \quad 1526(2013)$.

654 14. Kuhn, J. \& Dumont, S. Spindle assembly checkpoint satisfaction occurs via end-on but 655 not lateral attachments under tension. J. Cell Biol. 216, 1533-1542 (2017).

656 15. Wood, K. W. et al. Antitumor activity of an allosteric inhibitor of centromere-associated

657 protein-E. Proc. Natl. Acad. Sci. U. S. A. 107, 5839-5844 (2010).

658 16. Weihua, Z., Lin, Q., Ramoth, A. J., Fan, D. \& Fidler, I. J. Formation of solid tumors by a 659 single multinucleated cancer cell. Cancer 117, 4092-4099 (2011).

660 17. Mirzayans, R., Andrais, B. \& Murray, D. Roles of Polyploid/Multinucleated Giant Cancer 661 Cells in Metastasis and Disease Relapse Following Anticancer Treatment. Cancers 10, 662 (2018).

663 18. Muller, P. A. J. \& Vousden, K. H. p53 mutations in cancer. Nat. Cell Biol. 15, 2-8 (2013).

664 19. Fujiwara, T. et al. Cytokinesis failure generating tetraploids promotes tumorigenesis in 665 p53-null cells. Nature 437, 1043-1047 (2005).

666 20. Thompson, S. L. \& Compton, D. A. Proliferation of aneuploid human cells is limited by a 667 p53-dependent mechanism. J. Cell Biol. 188, 369-381 (2010).

668 21. Santaguida, S. et al. Chromosome Mis-segregation Generates Cell-Cycle-Arrested 669 Cells with Complex Karyotypes that Are Eliminated by the Immune System. Dev. Cell

$670 \quad 41,638-651 . e 5$ (2017).

671 22. Liu, S. et al. Nuclear envelope assembly defects link mitotic errors to chromothripsis.

$672 \quad$ Nature 561, 551-555 (2018). 
673 23. Escribano-Dıaz, C., Orthwein, A., Fradet-Turcotte, A., Xing, M. \& Young, J. T. Tká c J, 674 Cook MA, Rosebrock AP, Munro M, Canny MD, Xu D, Durocher D. 2013. A cell cycle675 dependent regulatory circuit composed of 53BP1-RIF1 and BRCA1-CtIP controls DNA 676 repair pathway choice. Mol Cell 49, 872-883.

677 24. Zimmermann, M., Lottersberger, F., Buonomo, S. B., Sfeir, A. \& de Lange, T. 53BP1

678 Regulates DSB Repair Using Rif1 to Control 5' End Resection. Science vol. 339700 $679704(2013)$.

680 25. Chapman, J. R. et al. RIF1 is essential for 53BP1-dependent nonhomologous end 681 joining and suppression of DNA double-strand break resection. Mol. Cell 49, 858-871 $682 \quad$ (2013).

683 26. Harrigan, J. A. et al. Replication stress induces 53BP1-containing OPT domains in G1 684 cells. J. Cell Biol. 193, 97-108 (2011).

685 27. Heldt, F. S., Barr, A. R. \& Cooper, S. A comprehensive model for the proliferation686 quiescence decision in response to endogenous DNA damage in human cells. $687 \quad$ Proceedings of the (2018).

688 28. Reyes, M. E. et al. RNA sequence analysis of inducible pluripotent stem cell-derived 689 cardiomyocytes reveals altered expression of DNA damage and cell cycle genes in 690 response to doxorubicin. Toxicol. Appl. Pharmacol. 356, 44-53 (2018).

691 29. Barr, A. R. et al. DNA damage during S-phase mediates the proliferation-quiescence 692 decision in the subsequent G1 via p21 expression. Nature Communications vol. 8 $693 \quad$ (2017).

694 30. Spencer, S. L. et al. The proliferation-quiescence decision is controlled by a bifurcation 695 in CDK2 activity at mitotic exit. Cell 155, 369-383 (2013).

696 31. Gascoigne, K. E. \& Taylor, S. S. Cancer cells display profound intra- and interline 697 variation following prolonged exposure to antimitotic drugs. Cancer Cell 14, 111-122 $698 \quad(2008)$.

699 32. Orth, J. D. et al. Quantitative live imaging of cancer and normal cells treated with 700 Kinesin-5 inhibitors indicates significant differences in phenotypic responses and cell 
701 fate. Mol. Cancer Ther. 7, 3480-3489 (2008).

702 33. Shi, J., Orth, J. D. \& Mitchison, T. Cell Type Variation in Responses to Antimitotic Drugs

703 that Target Microtubules and Kinesin-5. Cancer Research vol. 68 3269-3276 (2008).

704 34. Uetake, Y. \& Sluder, G. Prolonged Prometaphase Blocks Daughter Cell Proliferation

705 Despite Normal Completion of Mitosis. Current Biology vol. 20 1666-1671 (2010).

706 35. Orth, J. D., Loewer, A., Lahav, G. \& Mitchison, T. J. Prolonged mitotic arrest triggers

707 partial activation of apoptosis, resulting in DNA damage and p53 induction. Mol. Biol.

708 Cell 23, 567-576 (2012).

709 36. Fong, C. S. et al. 53BP1 and USP28 mediate p53-dependent cell cycle arrest in

710 response to centrosome loss and prolonged mitosis. Elife 5, (2016).

711 37. Dalton, W. B., Nandan, M. O., Moore, R. T. \& Yang, V. W. Human cancer cells

712 commonly acquire DNA damage during mitotic arrest. Cancer Res. 67, 11487-11492

$713 \quad$ (2007).

714 38. Dobles, M., Liberal, V., Scott, M. L., Benezra, R. \& Sorger, P. K. Chromosome

715 Missegregation and Apoptosis in Mice Lacking the Mitotic Checkpoint Protein Mad2.

716 Cell vol. $101635-645$ (2000).

717 39. Rodier, F. et al. DNA-SCARS: distinct nuclear structures that sustain damage-induced

718 senescence growth arrest and inflammatory cytokine secretion. J. Cell Sci. 124, 68-81

719 (2011).

720 40. Mackenzie, K. J. et al. cGAS surveillance of micronuclei links genome instability to

721 innate immunity. Nature 548, 461-465 (2017).

722 41. Harding, S. M. et al. Mitotic progression following DNA damage enables pattern 723 recognition within micronuclei. Nature 548, 466-470 (2017).

724 42. Lele, T. P., Dickinson, R. B. \& Gundersen, G. G. Mechanical principles of nuclear 725 shaping and positioning. J. Cell Biol. 217, 3330-3342 (2018).

726 43. Lovett, D. B., Shekhar, N., Nickerson, J. A., Roux, K. J. \& Lele, T. P. Modulation of

$727 \quad$ Nuclear Shape by Substrate Rigidity. Cell. Mol. Bioeng. 6, 230-238 (2013).

728 44. Worman, H. J. \& Bonne, G. 'Laminopathies': A wide spectrum of human diseases. 
bioRxiv preprint doi: https://doi.org/10.1101/2020.12.22.424035; this version posted December 22, 2020. The copyright holder for this preprint (which was not certified by peer review) is the author/funder, who has granted bioRxiv a license to display the preprint in perpetuity. It is made available under aCC-BY-ND 4.0 International license. 
bioRxiv preprint doi: $\mathrm{https}$ //doi.org/10.1101/2020.12.22.424035; this version posted December 22, 2020. The copyright holder for this preprint (which was not certified by peer review) is the author/funder, who has granted bioRxiv a license to display the preprint in perpetuity. It is made available under aCC-BY-ND 4.0 International license.

732 
A

bioRxiv preprint doi: https://doi.org/10.1101/2020.12.22.424035; this version posted December 22, 2020. The copyright holder for this preprint (which was not certified by peer review) is the author/funder, who has granted bioRxiv a license to display the preprint in perpetuity. It is made RPE1 p53 WT/

RPE1 p53 k.d. cells

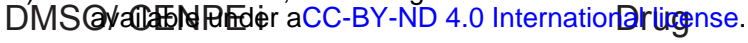

Fixation and

treatment

washout

Immunostain

$-16 h$

Oh

48h
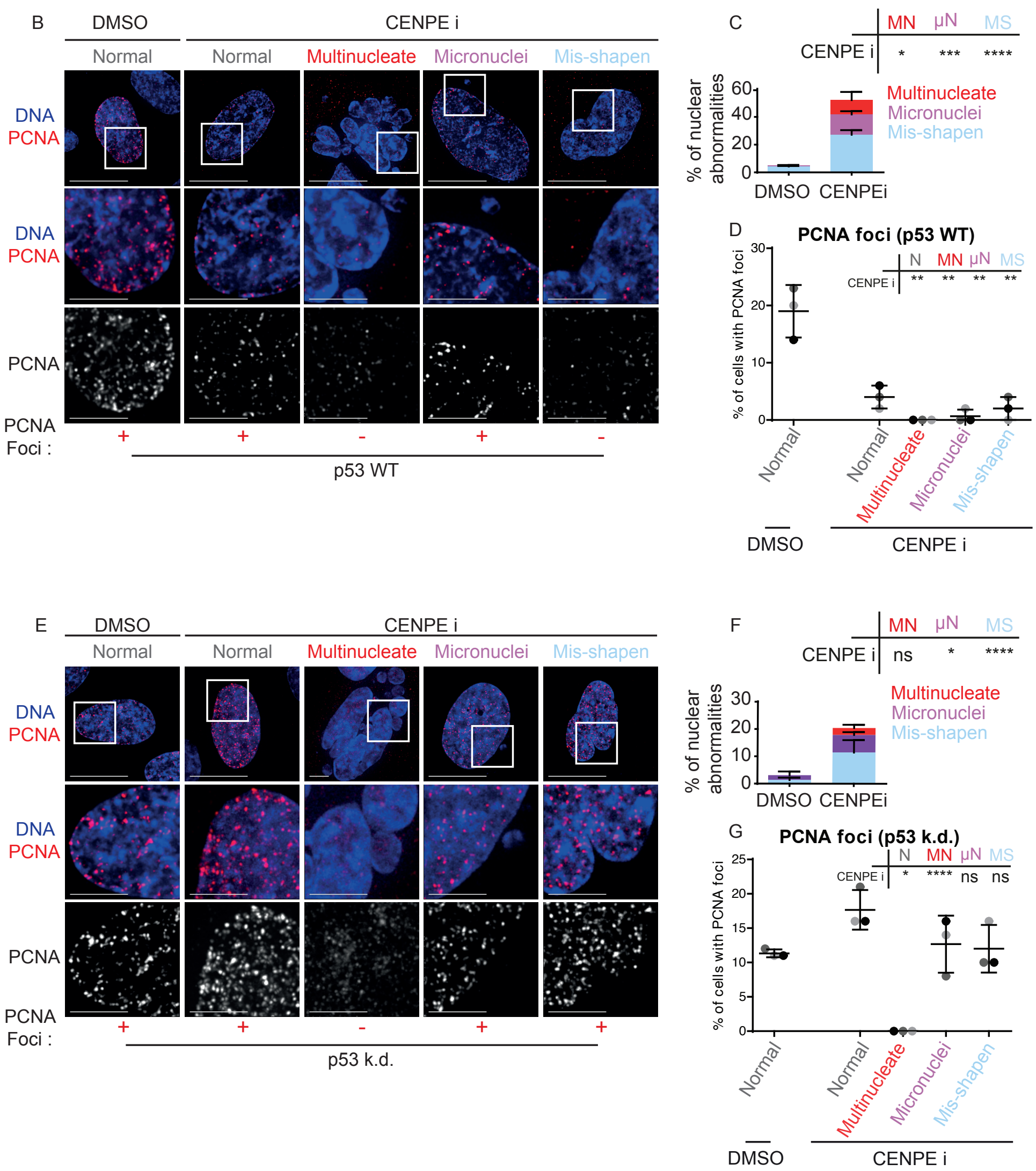
bioRxiv preprint doi: https://doi.org/10.1101/2020.12.22.424035; this version posted December 22, 2020. The copyright holder for this preprint (which was not certified by peer review) is the author/funder, who has granted bioRxiv a license to display the preprint in perpetuity. It is made

A

$$
\text { DMSO }
$$
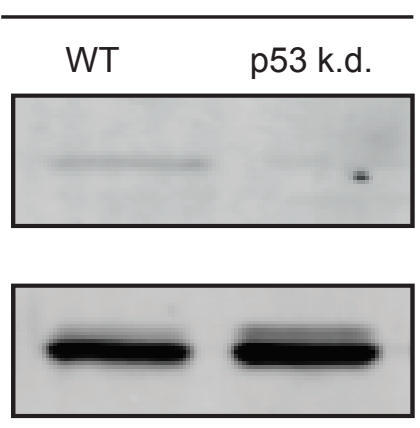

avaidebljpend

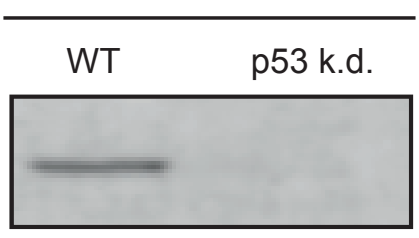

p53

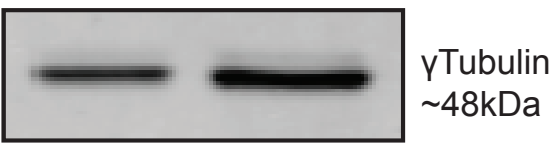

$$
\text { B license. }
$$

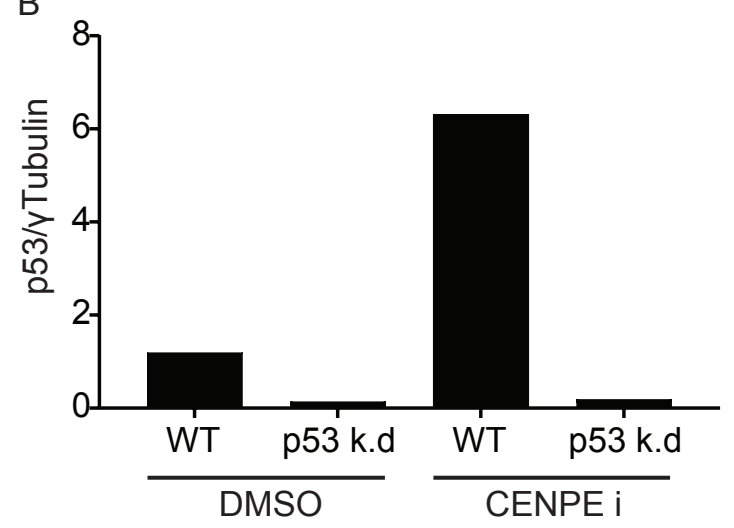




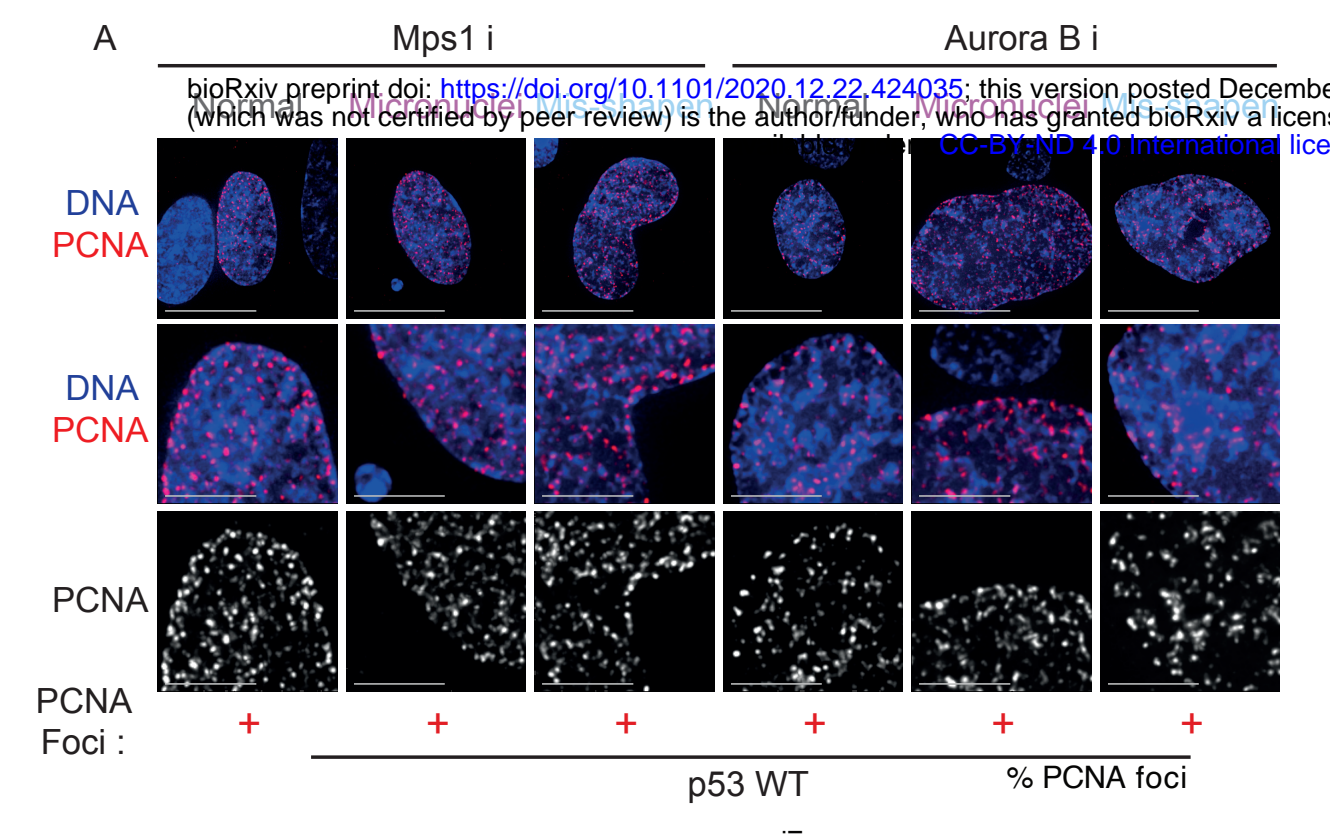

B

\begin{tabular}{l|l} 
& MN $\mu N \quad M S$ \\
\hline The copyrigntgoildiel fosthis preprint***
\end{tabular}

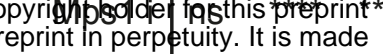

Aurora B i ns ${ }^{* * * *}{ }^{* * * *}$

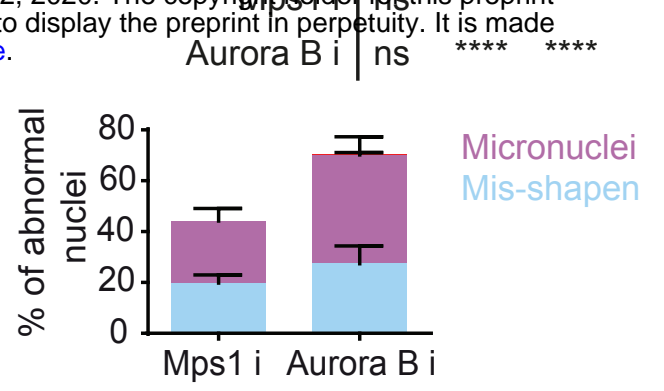

\begin{tabular}{lr|cccc} 
C & N & MN & $\mu N$ & MS \\
\hline Mps1 i & ns & na & $*$ & $*$ \\
Aurora B i & $*$ & na & $*$ & $*$
\end{tabular}
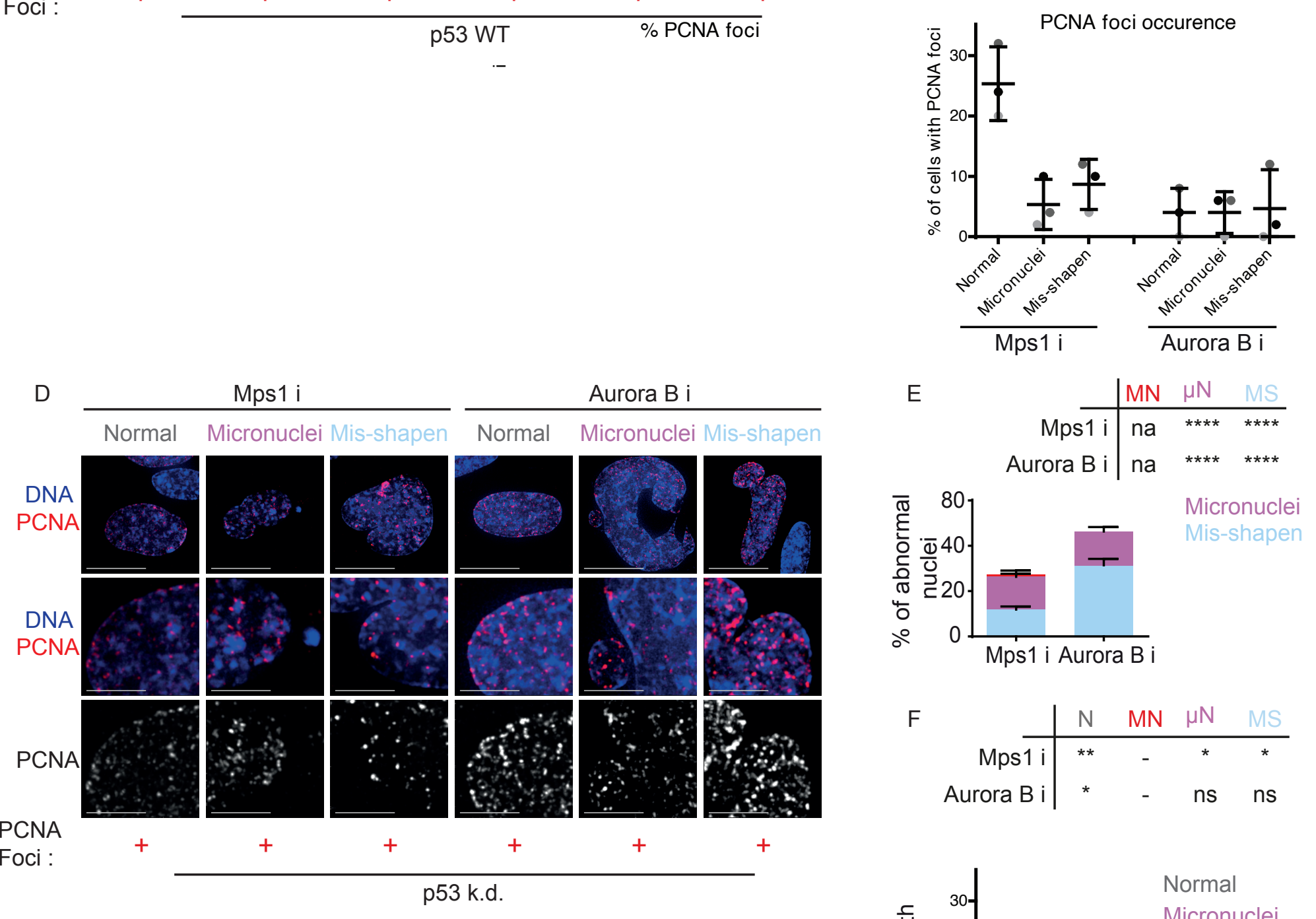

\begin{tabular}{lr|cccc}
$\mathrm{F}$ & $\mathrm{N}$ & $\mathrm{MN}$ & $\mu \mathrm{N}$ & $\mathrm{MS}$ \\
\hline & $\mathrm{Mps} 1 \mathrm{i}$ & $* *$ & - & $*$ & $*$ \\
Aurora B i & $*$ & - & $\mathrm{ns}$ & $\mathrm{ns}$
\end{tabular}

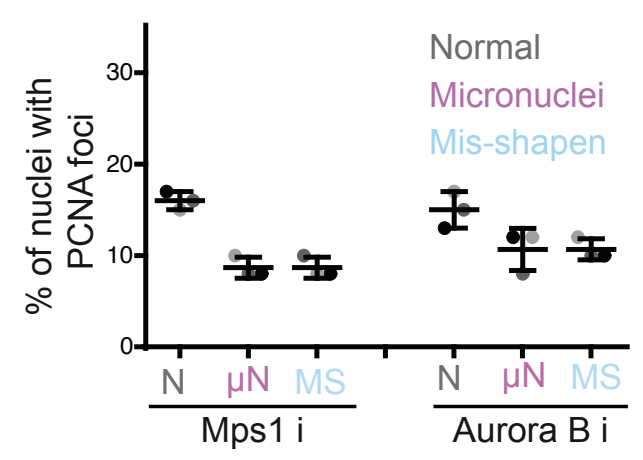




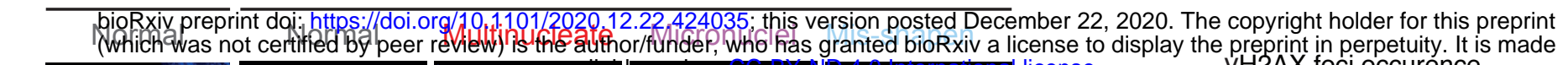
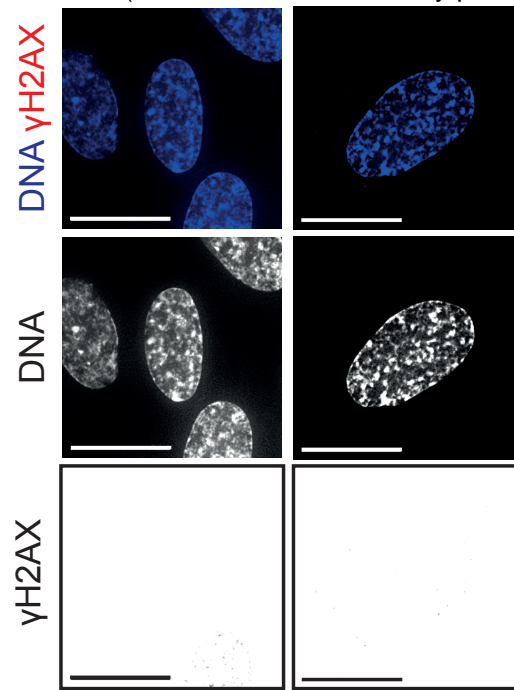

C

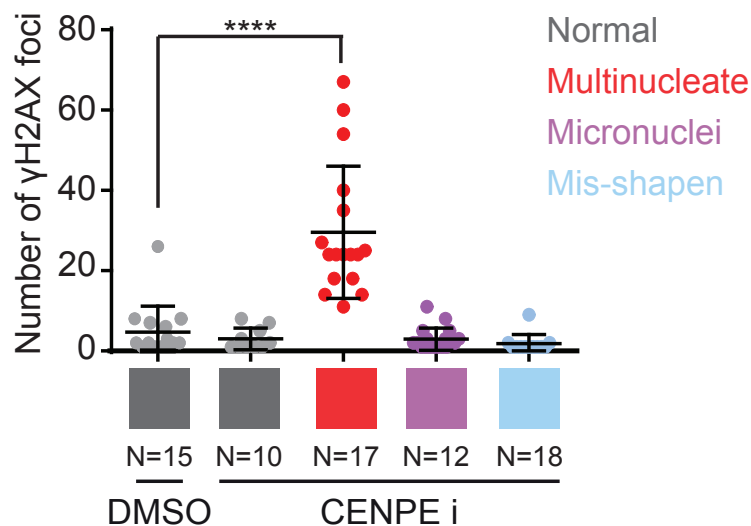

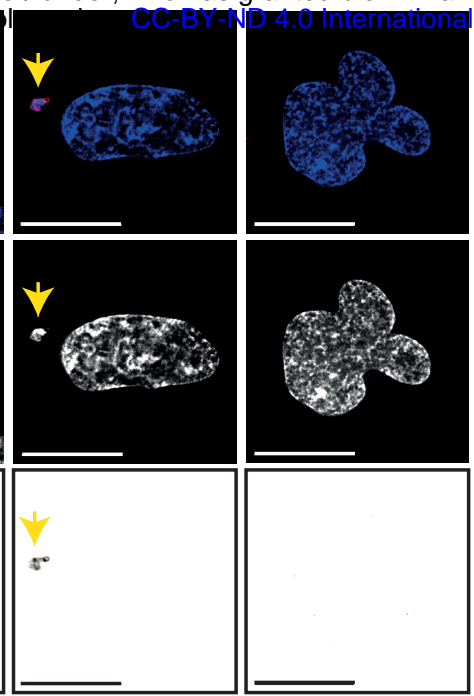

Scale bar $15 \mu \mathrm{m}$

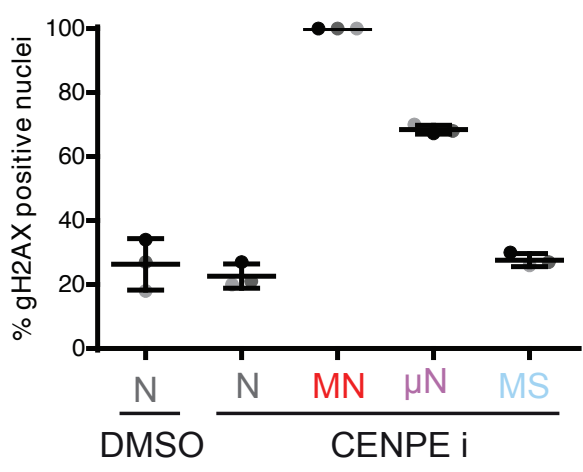

\begin{tabular}{c|cccc} 
& $\mathrm{N}$ & $\mathrm{MN}$ & $\mu \mathrm{N}$ & $\mathrm{MS}$ \\
\hline CENPE i & $\mathrm{ns}$ & $* * * *$ & $* * *$ & $\mathrm{~ns}$
\end{tabular}

F DMSO CENPE i Aphi
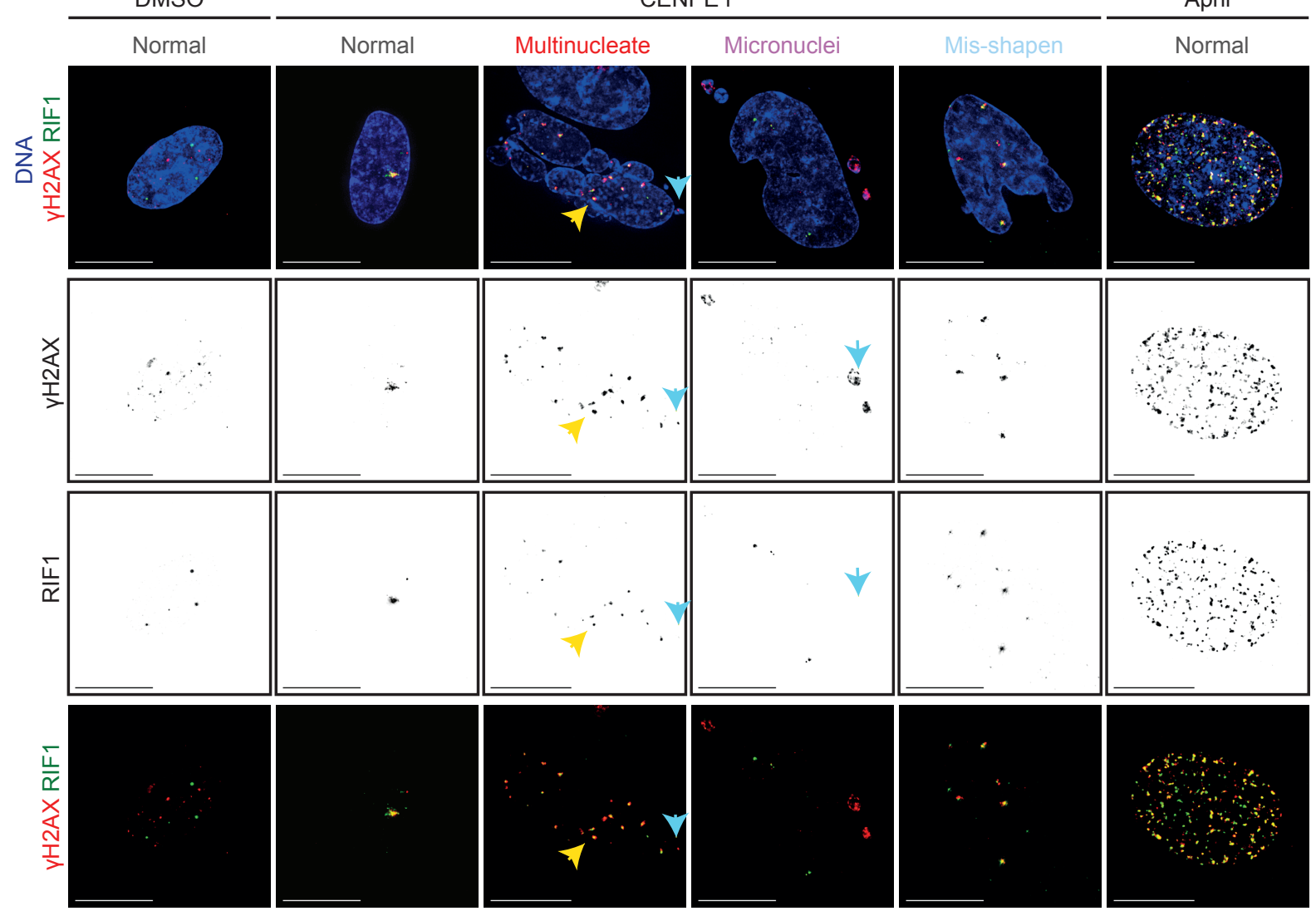

Scale bar $15 \mu \mathrm{m}$ 


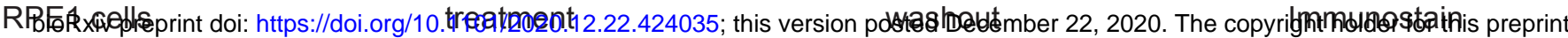
(which was not certified by peer review) is the author/funder, who has granted bioRyiv a license to display the preprint in perpetuity. It is made

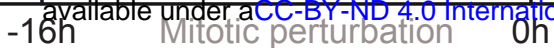

$1 \mathrm{~h}$

B

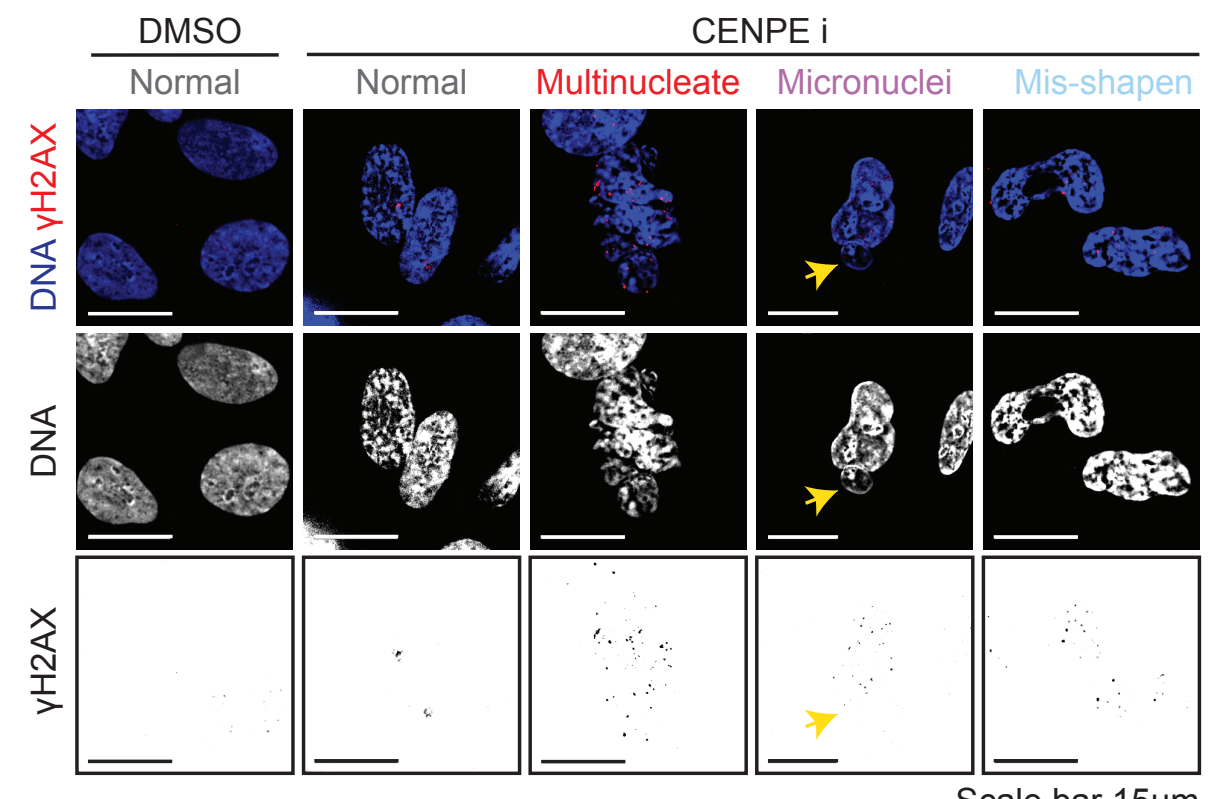

Scale bar $15 \mu \mathrm{m}$
C - Proportion of abnormal nuclei

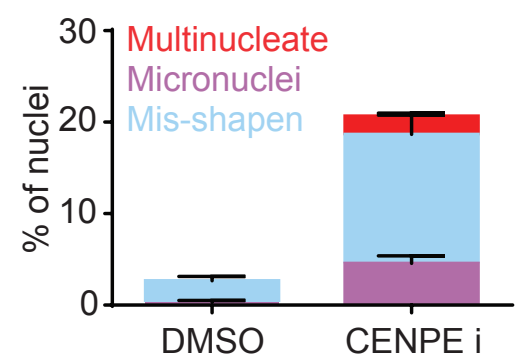

E - Number of $\mathrm{yH} 2 \mathrm{AX}$ foci per nucleus

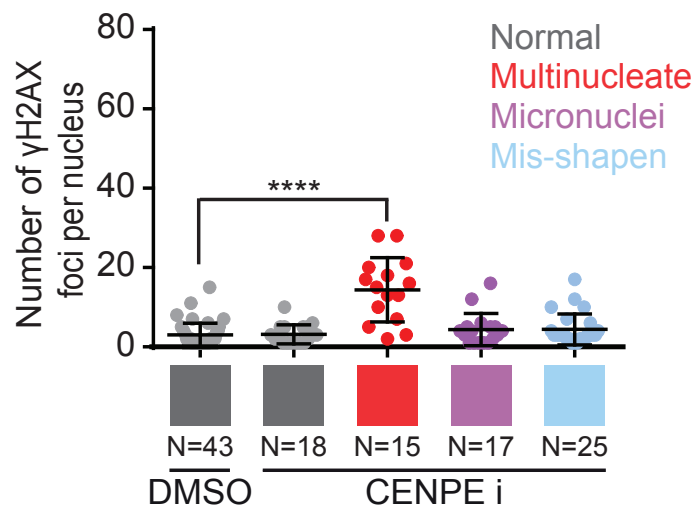

Multinucleate

Micronuclei

DIMSO CENPE I

F - Fluorescence intensity of $\mathrm{YH} 2 \mathrm{AX}$ foci

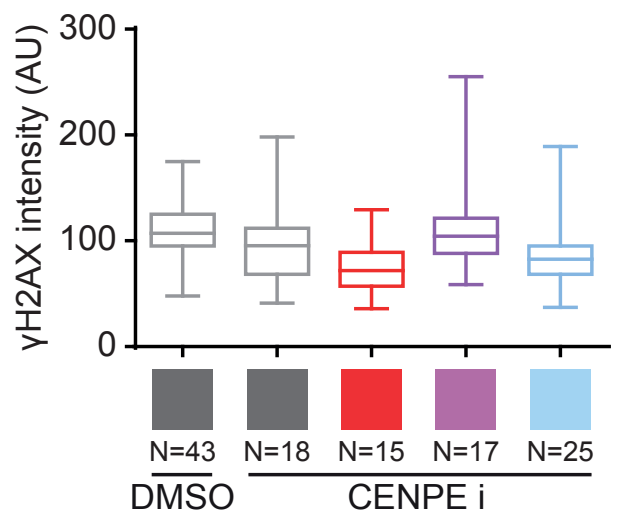

Normal

Multinucleate

Micronuclei

Mis-shapen

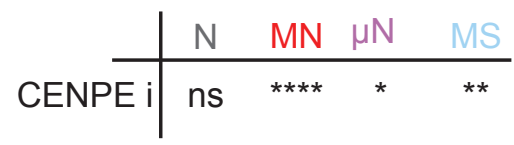

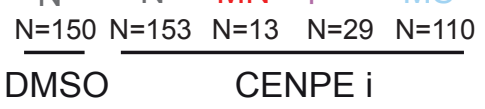

DMSO

CENPE i 
A bioRxiv preprint doi: https://doi.org/101101/2020.12.22.424035; this version posted December 22, 2020. The copyright holder for this preprint

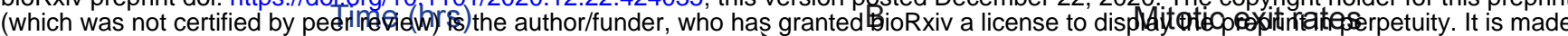
00:00 04:00 09:15 4.0 International license.

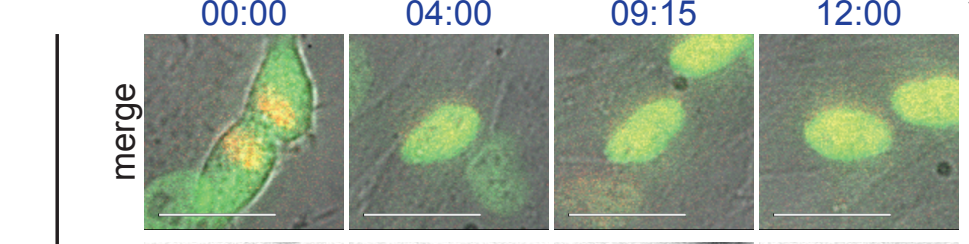

$\sum_{0}^{\circ}$

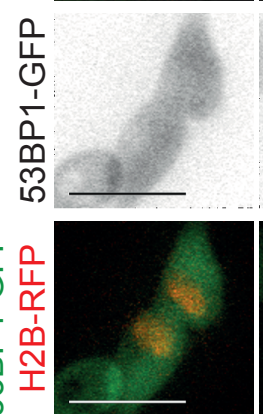

00:00

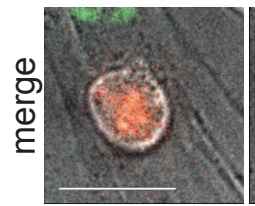

$01: 30$

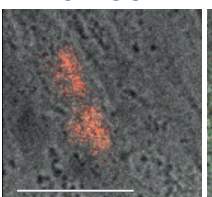

09:30

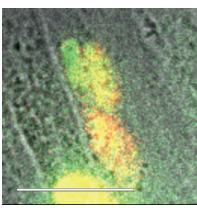

뜬

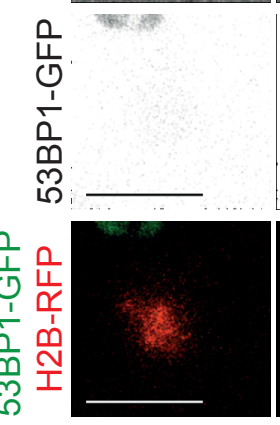

00:00

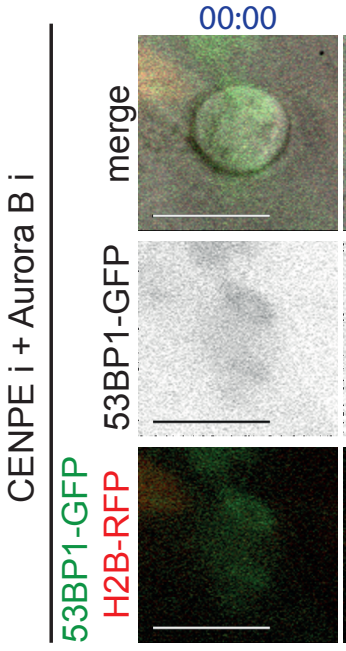

$02: 15$
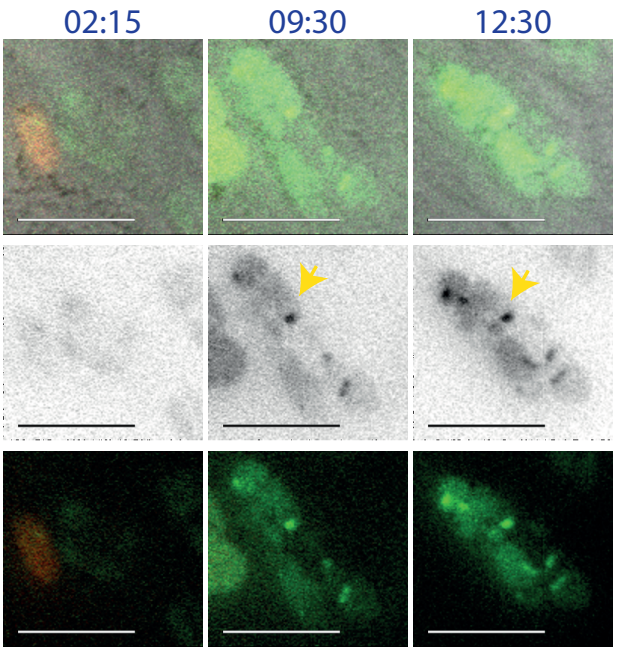

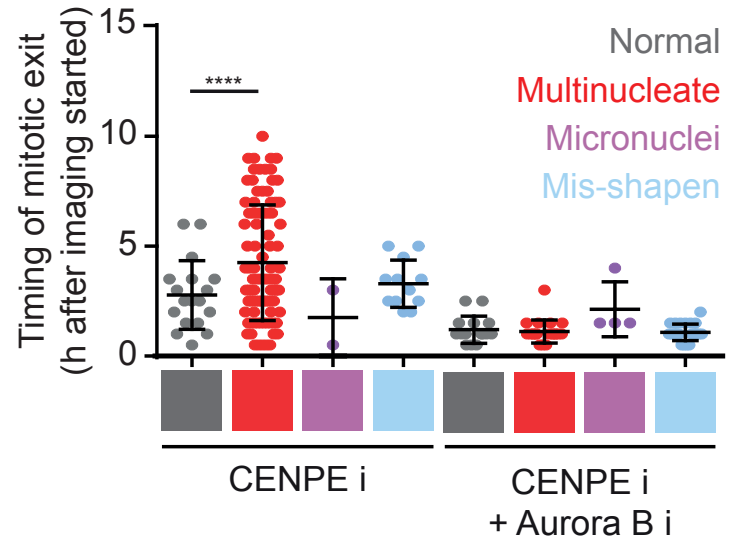

C

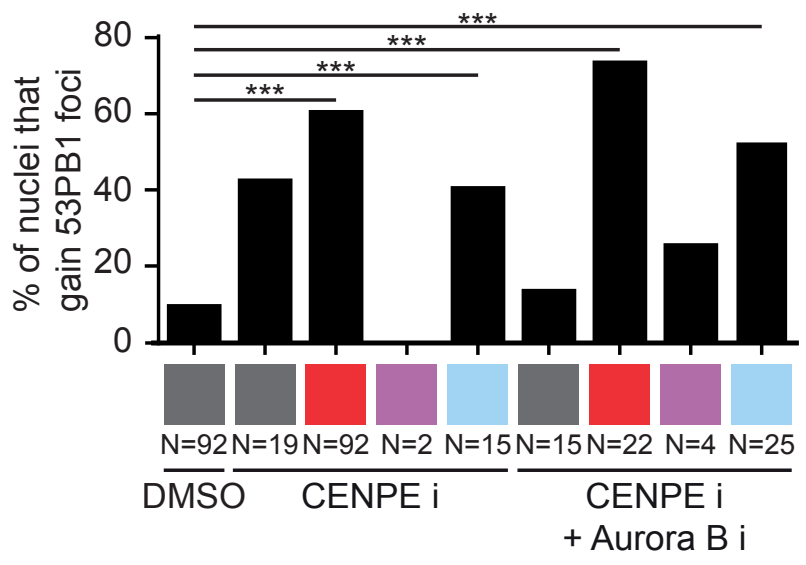

D

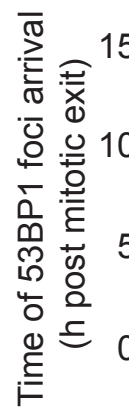

53BP1-foci arrival time

Normal

Multinucleate

Micronuclei

Mis-shaper

Temporal Change in 53BP1 Foci numbers

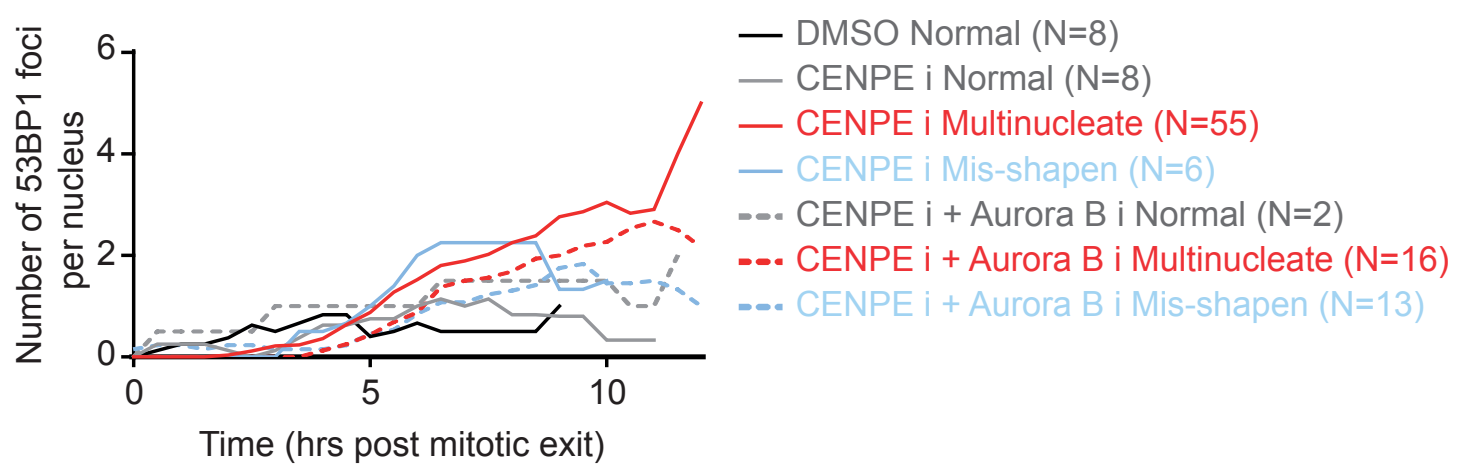


A

bioRxiv preprint doi: https://doi.org/10.1101/2020.12.22.424035; this version posted December 22, 2020. The copyright holder for this preprint

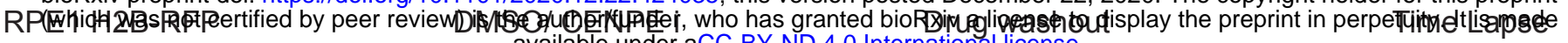
53BP1-GFP cells available ynder aCC-BY-ND 4.0 Internationablicense.ne well
treatrment Imaging
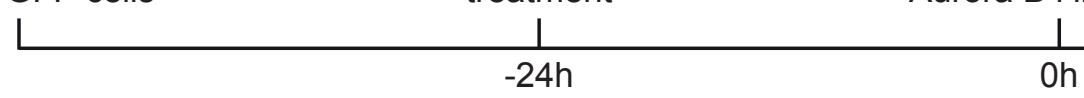
$\underset{\mathrm{Oh}}{\stackrel{\mathrm{H}}{\longrightarrow}}$

B

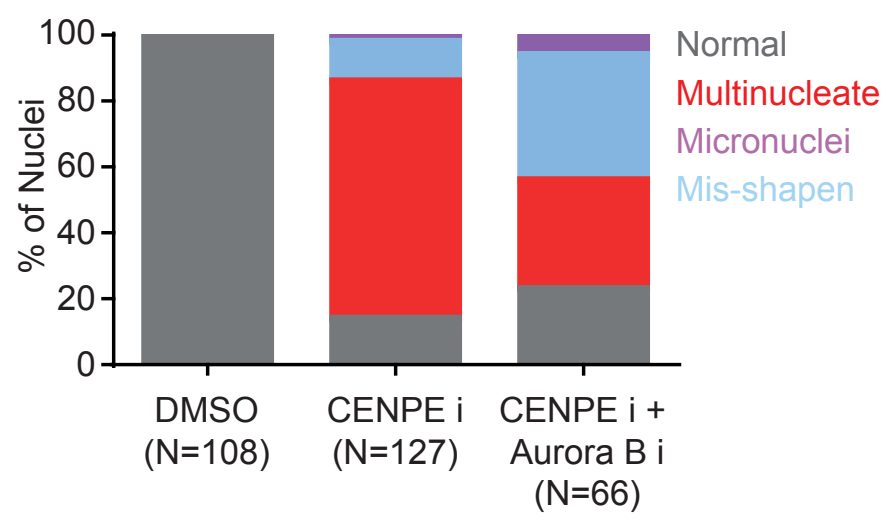

C

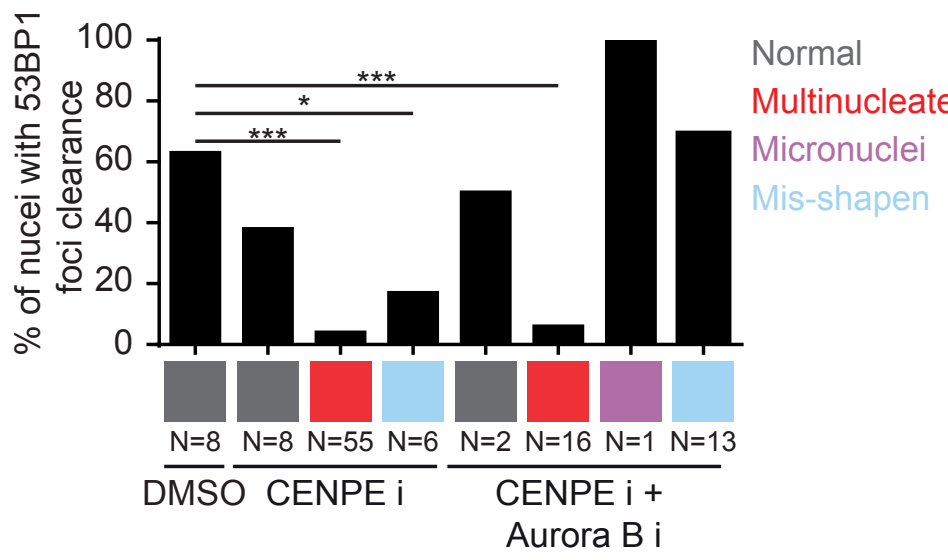

D - Representative cells showing number of 53BP1 foci following mitotic exit

\section{DMSO}

Time $(h)$

0.5

12.5

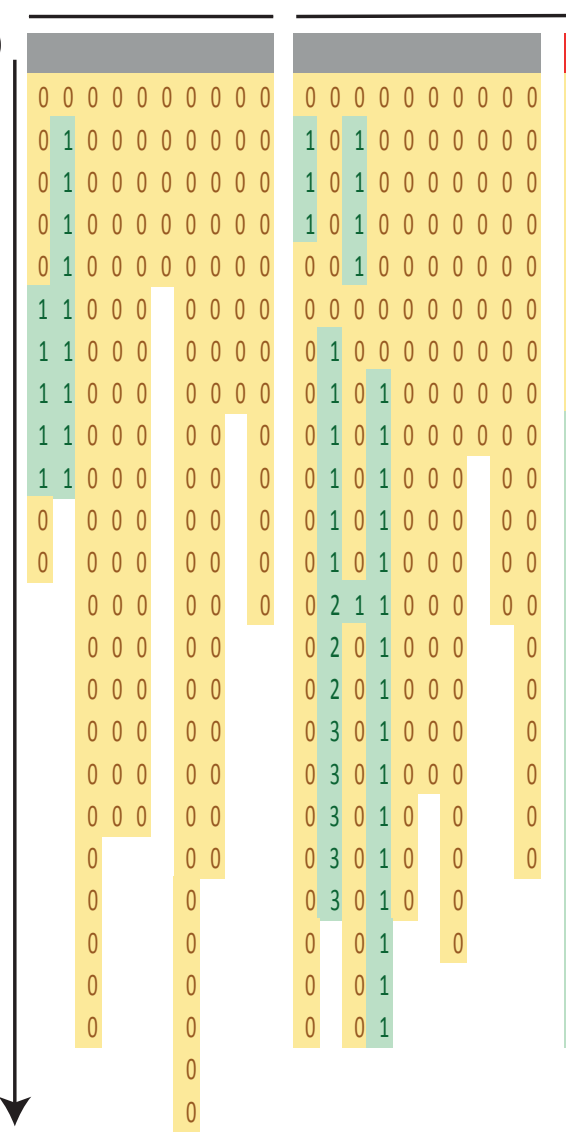

\section{CENPE i}

00000000000000 00000000000000 00000000000000 0000000000000 0000000000000 0000000000000 0000000000000 000000000000 1000000000000 30000000000000 3000000000000 3020000000000 3020020000000 $402202000 \quad 0$ 40220200 4240200 4240200 4240200

43440210 4441210 4441210 4441240 $\begin{array}{lll}5 & 4 & 0\end{array}$ $\begin{array}{ll}4 & 0 \\ & 0\end{array}$

\section{CENPE i + Aurora B i}

0000000000 000000000000 0000000000000 0000000000000 0000000000000 0000000000000 0000000000000 0000000000000 000000000000 00000000000 100000000000 1100000000

\section{0} 211000000 21100 $\begin{array}{lllll}2 & 1 & 1 & 0 & 0\end{array}$ 21100 21100 2110 211 21

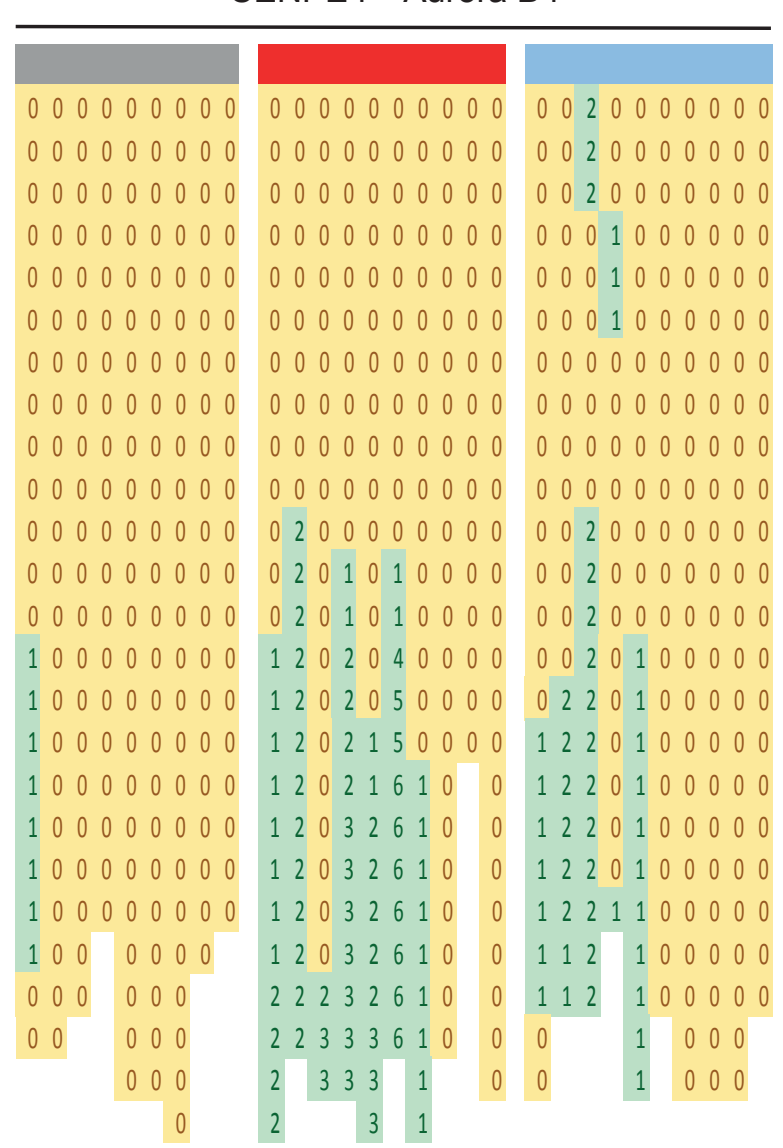


A RPE1 53BP1-GFP + CENPE i washout Time (hrs:mins)

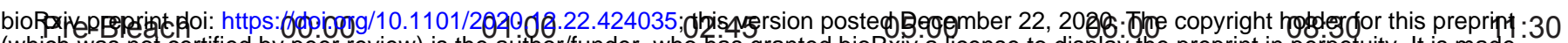
which was no certified by peer review) is the author/funder, who has granted bioBxiv a license to display the preprint in perpefuity. It is mado

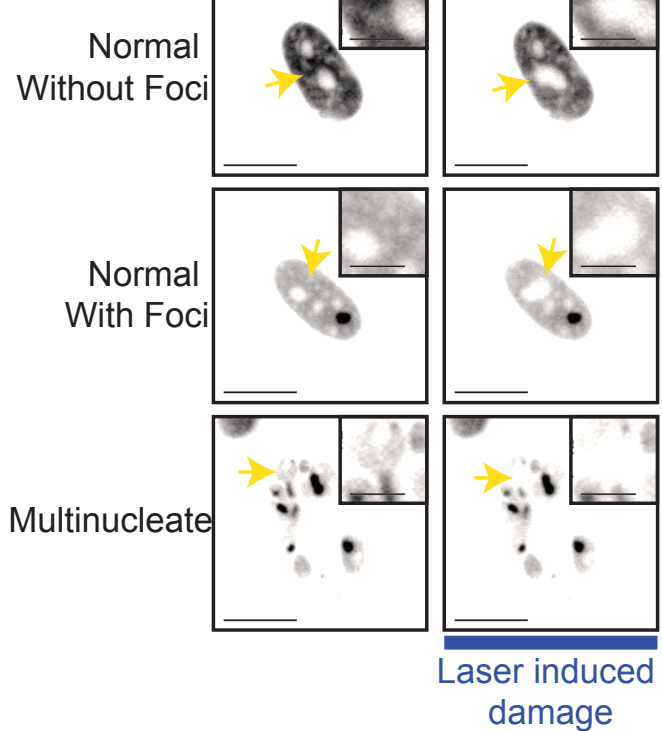

$B$ - Proportion of nuclei that gain laser induced 53BP1-GFP foci

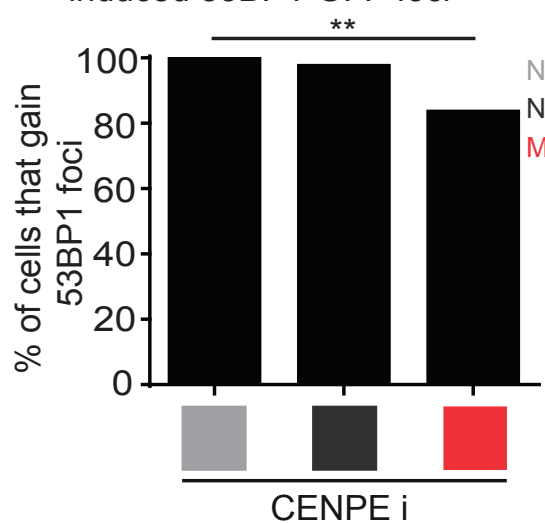

D - Proportion of nuclei that clear laser induced 53BP1-GFP foci

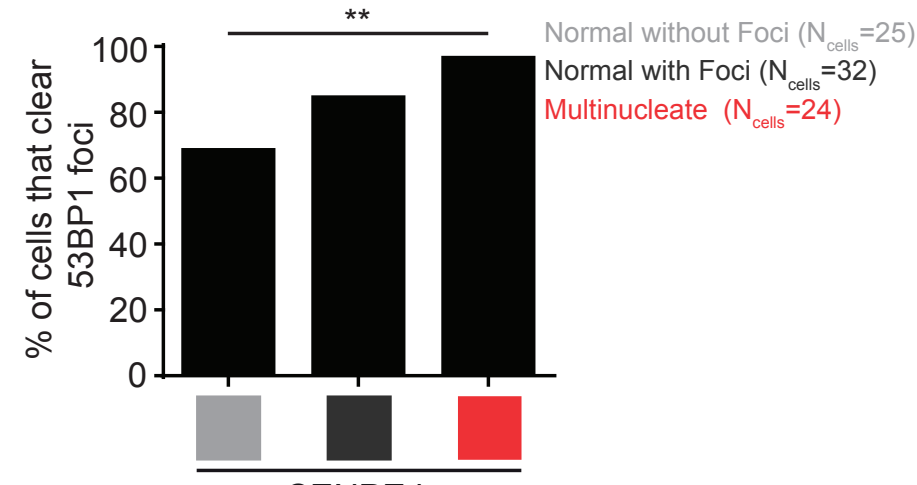

CENPE i

F - 53BP1-Foci intensity changes

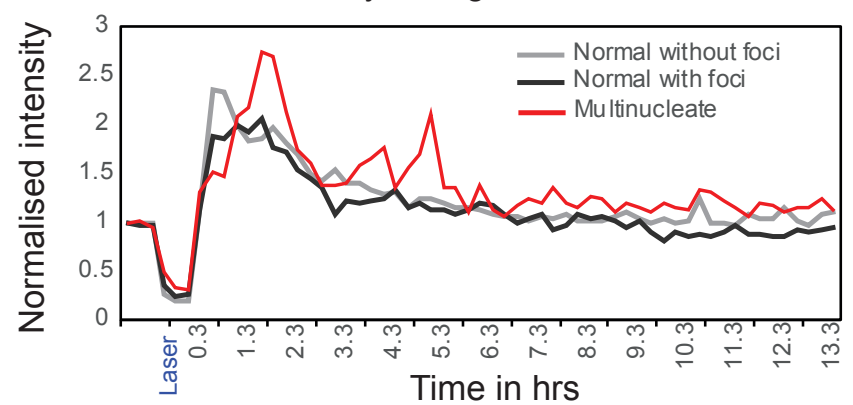

(Pre- \& Post- laser induction)

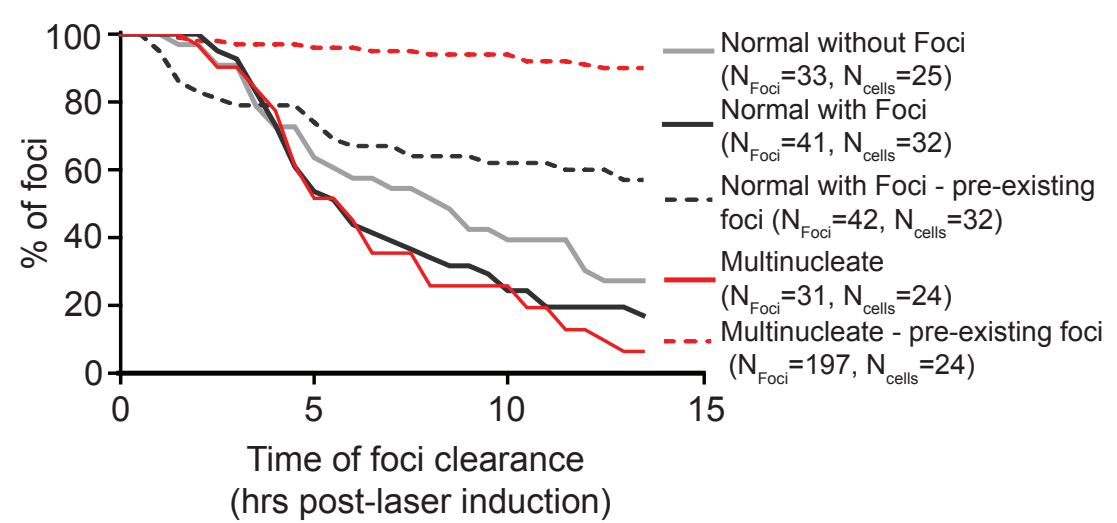

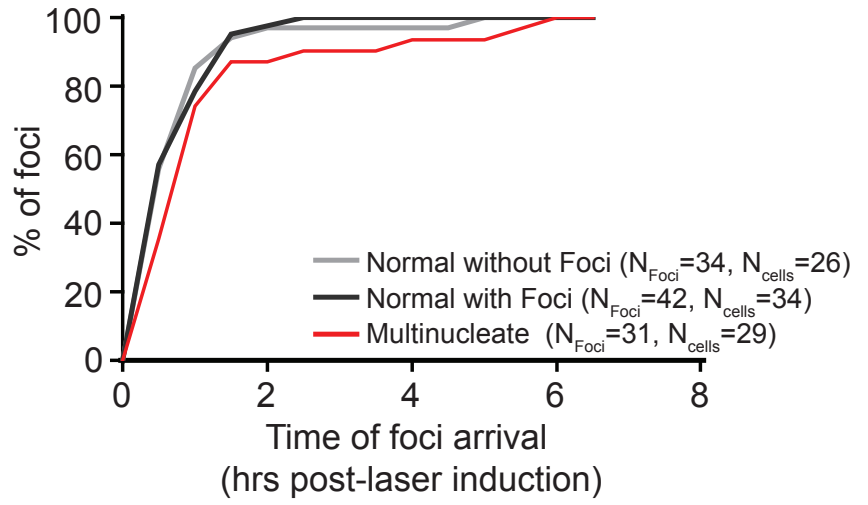

E - Timing of 53BP1-GFP foci clearance 

$\mathrm{hrs}$

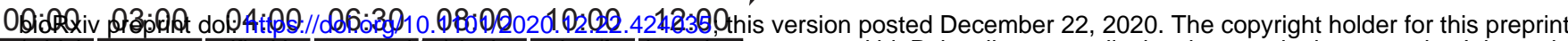

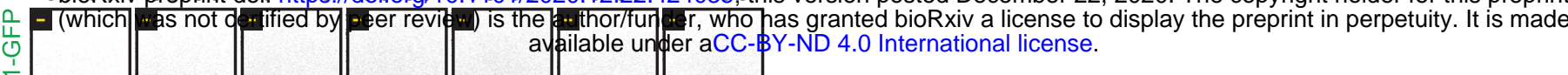

O
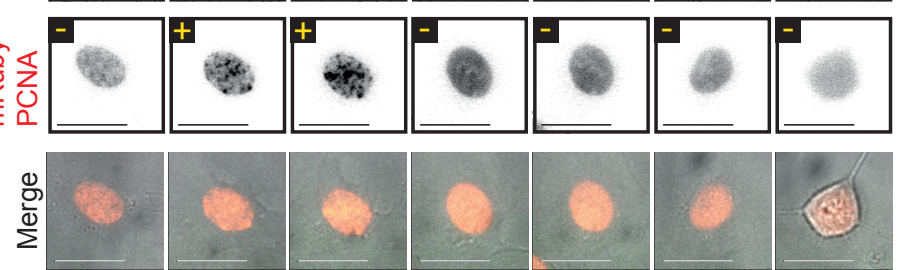

B

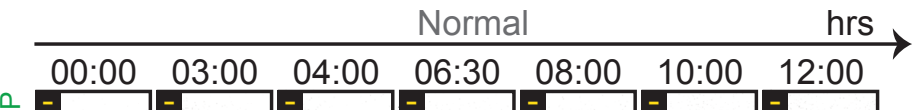
$\stackrel{\mathrm{hrs}}{\rightarrow}$
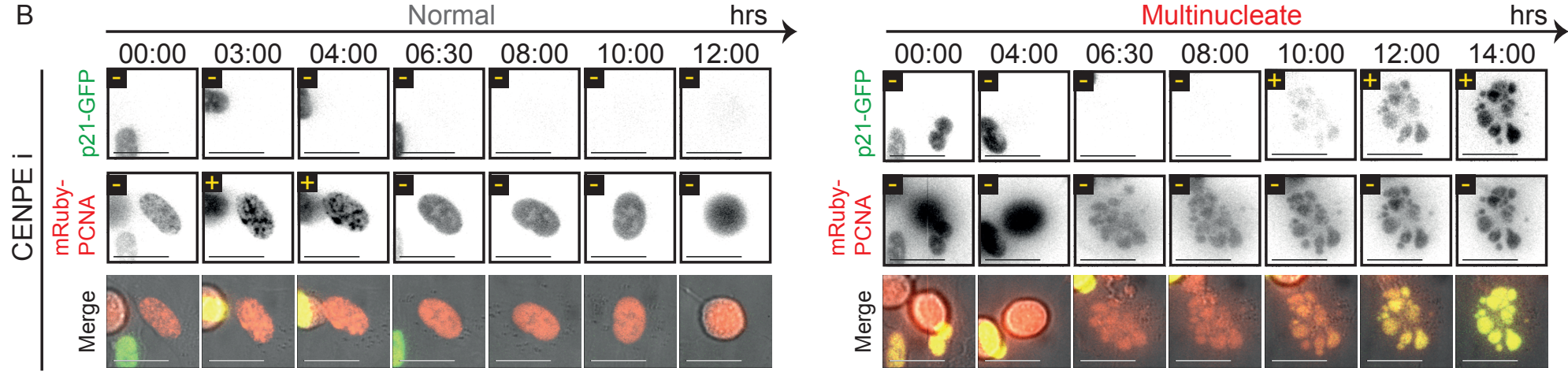

$c \bar{d}$

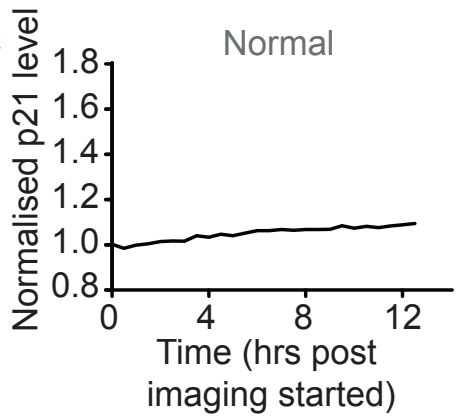

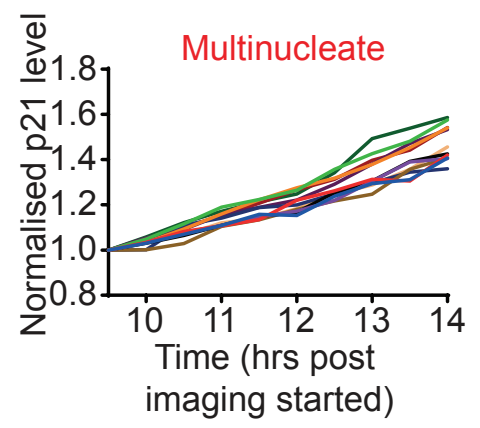

Normal

D

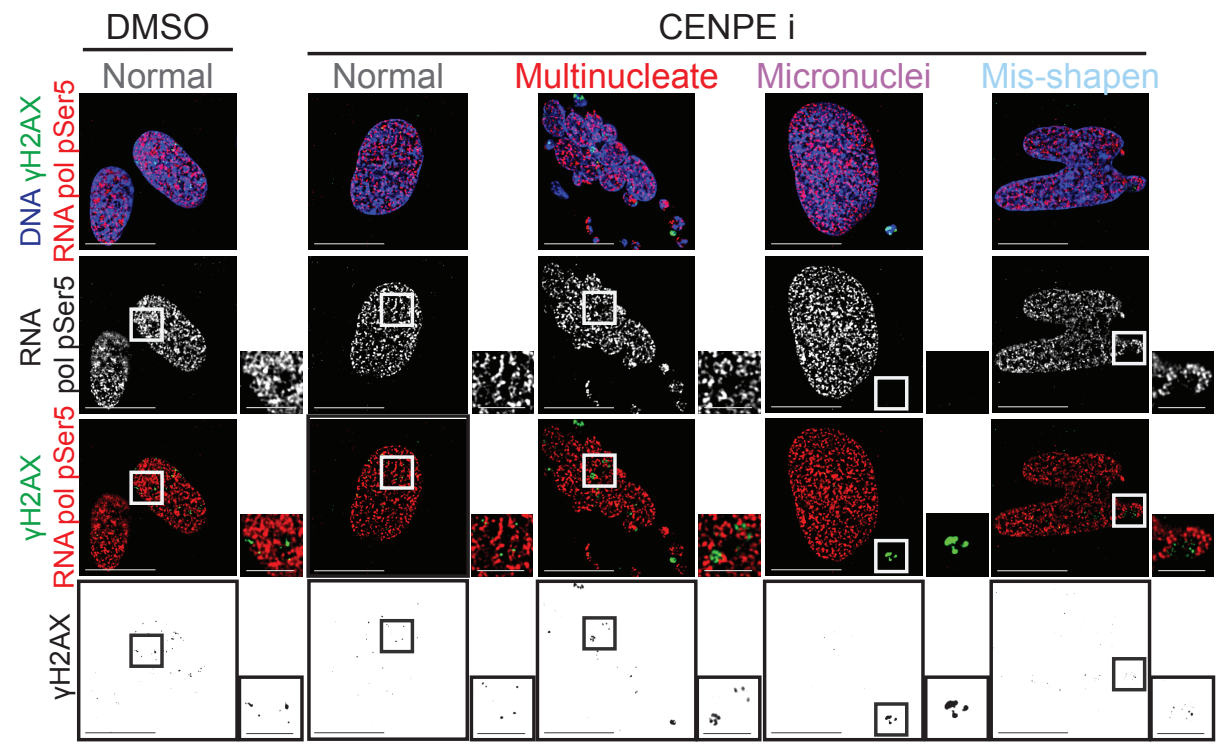

E

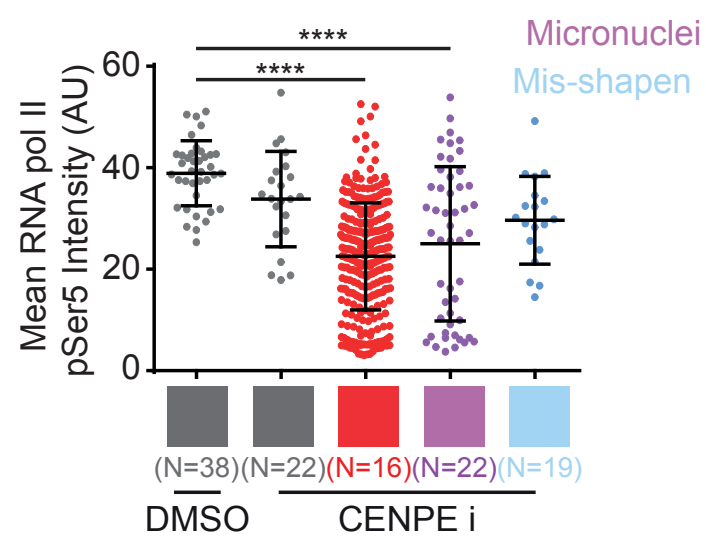

$\mathrm{F}$

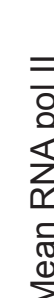

$$
\begin{aligned}
& =
\end{aligned}
$$

DMSOl Normal ( $\mathrm{N}=38)$

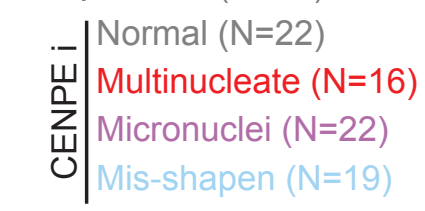

0

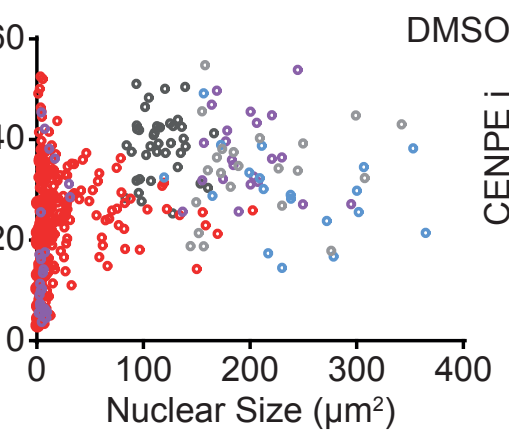


A

B

bioRxiv preprint doi: https://doi.org/10.1101/2020.12.22.424035; this version posted December 22, 2020. The copyright holder for this preprint (which was not certified by peer review) is the author/fundsegr, who has granted bioRxiv a license to display the preprint in perpetuity. It is made

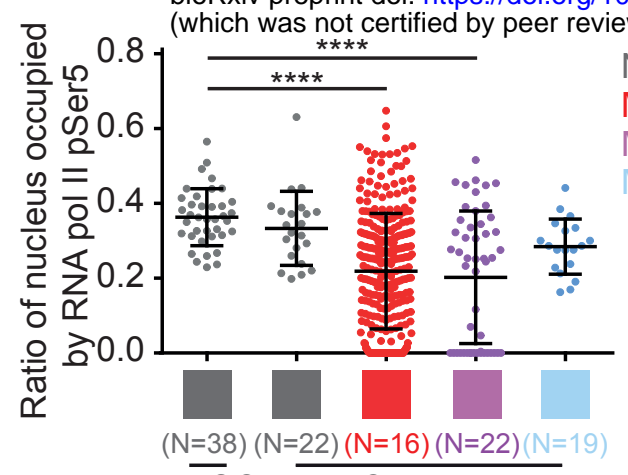

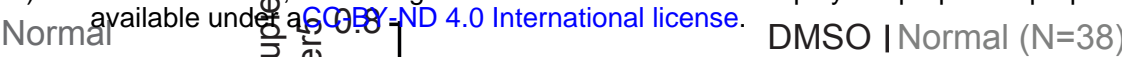

Multinucleate

Micronuclei

DMSO CENPE $\mathrm{i}$

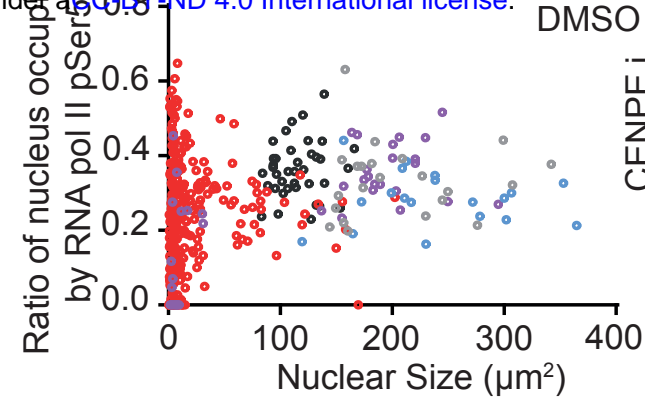

- Normal $(\mathrm{N}=22)$

Ш Multinucleate $(\mathrm{N}=16)$

Micronuclei $(\mathrm{N}=22)$

Mis-shapen ( $N=19)$
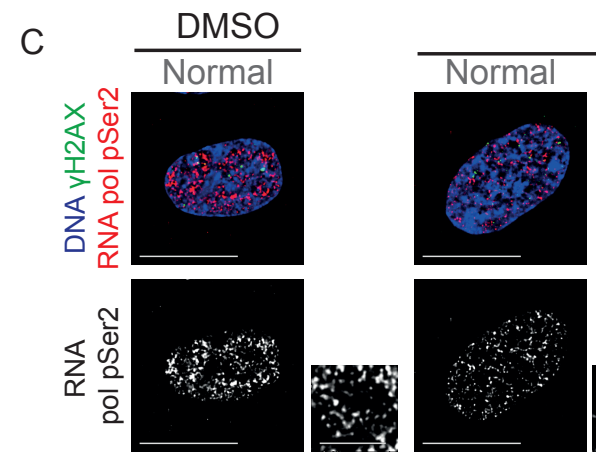

CENPE i
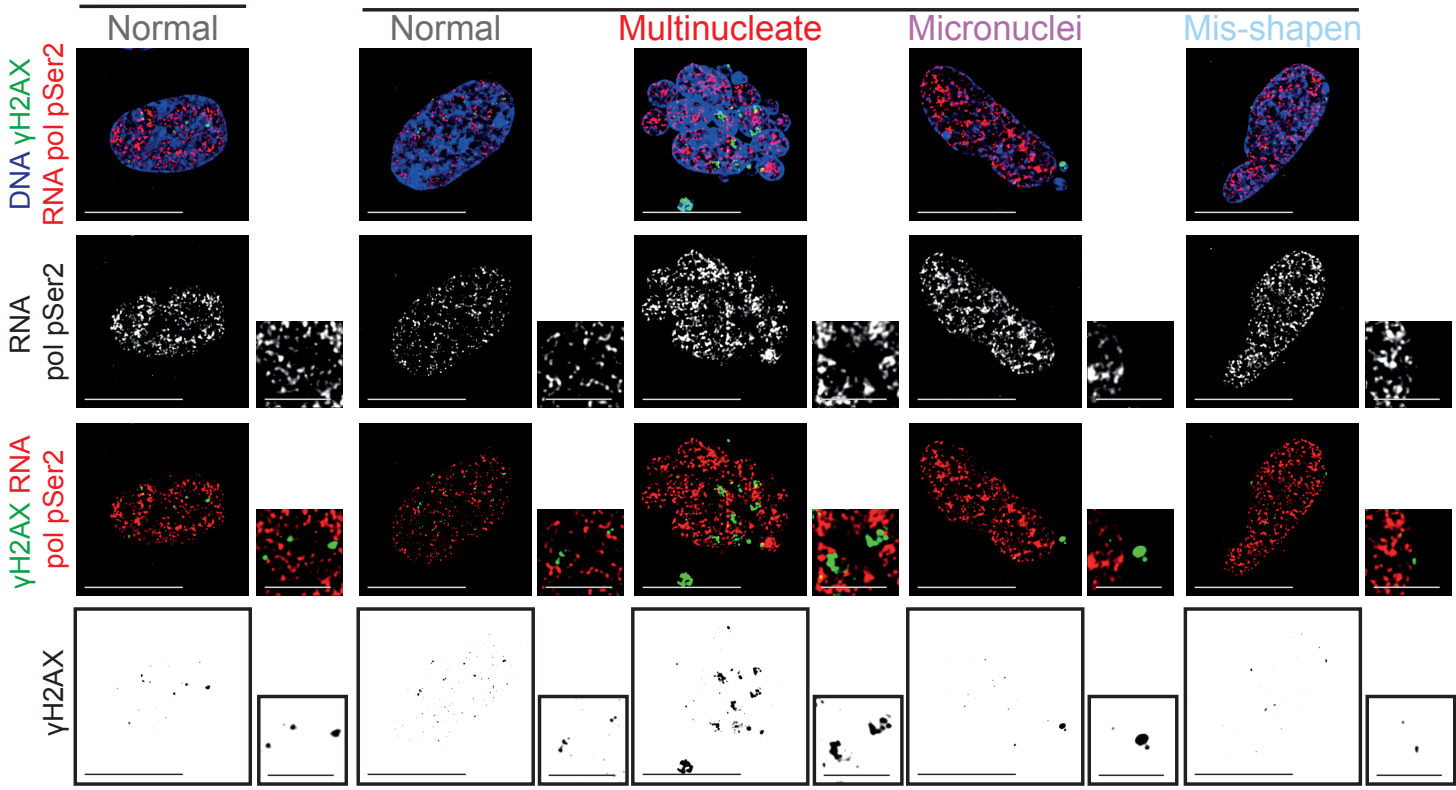

D - Mean RNA pol II CTD pSer2 intensity

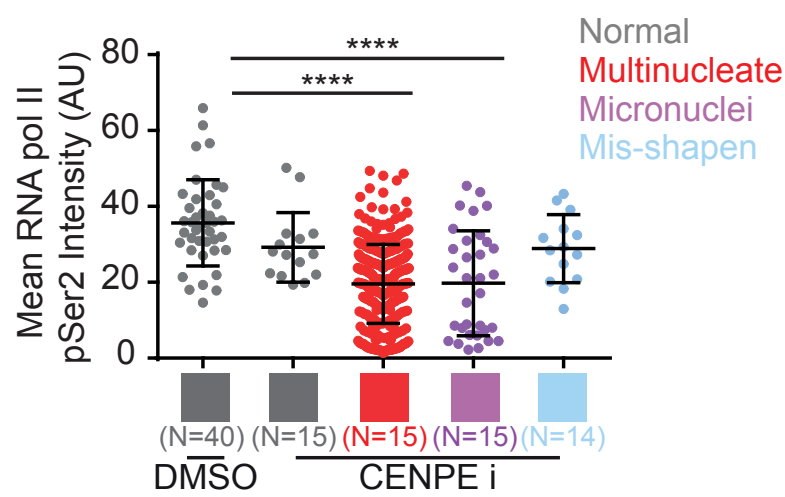

F - Proportion of nucleus occupied by RNA II CTD pSer2

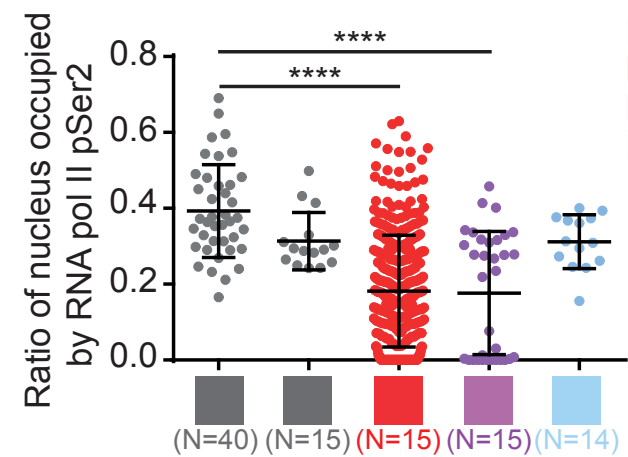

Normal

Multinucleate

Micronuclei

Mis-shapen
E - Mean RNA pol II CTD pSer2 inensity against size

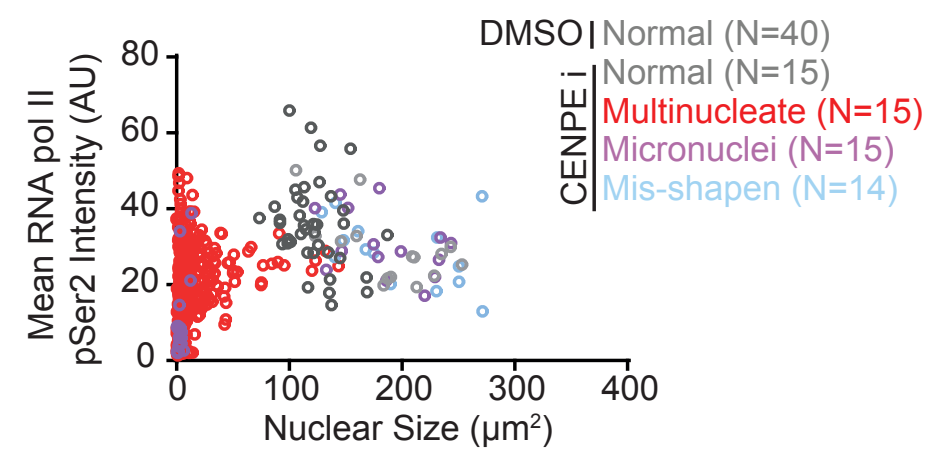

G - Proportion of nucleus occupied by RNA II CTD pSer2 against size

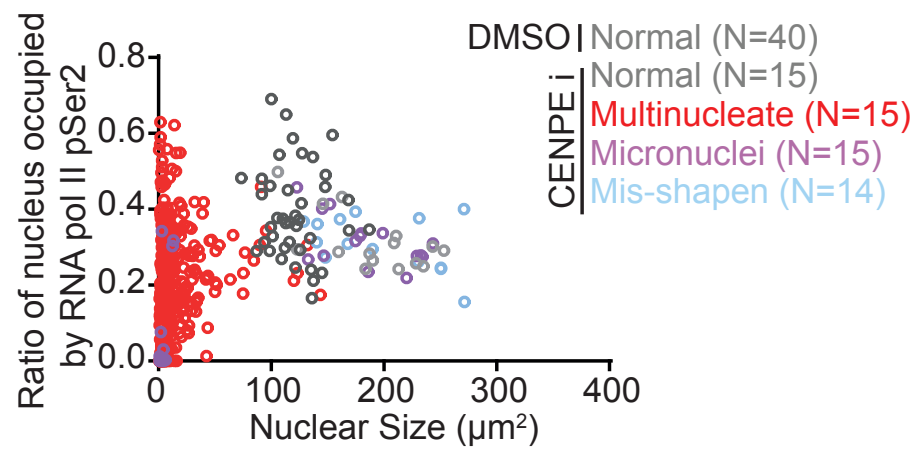



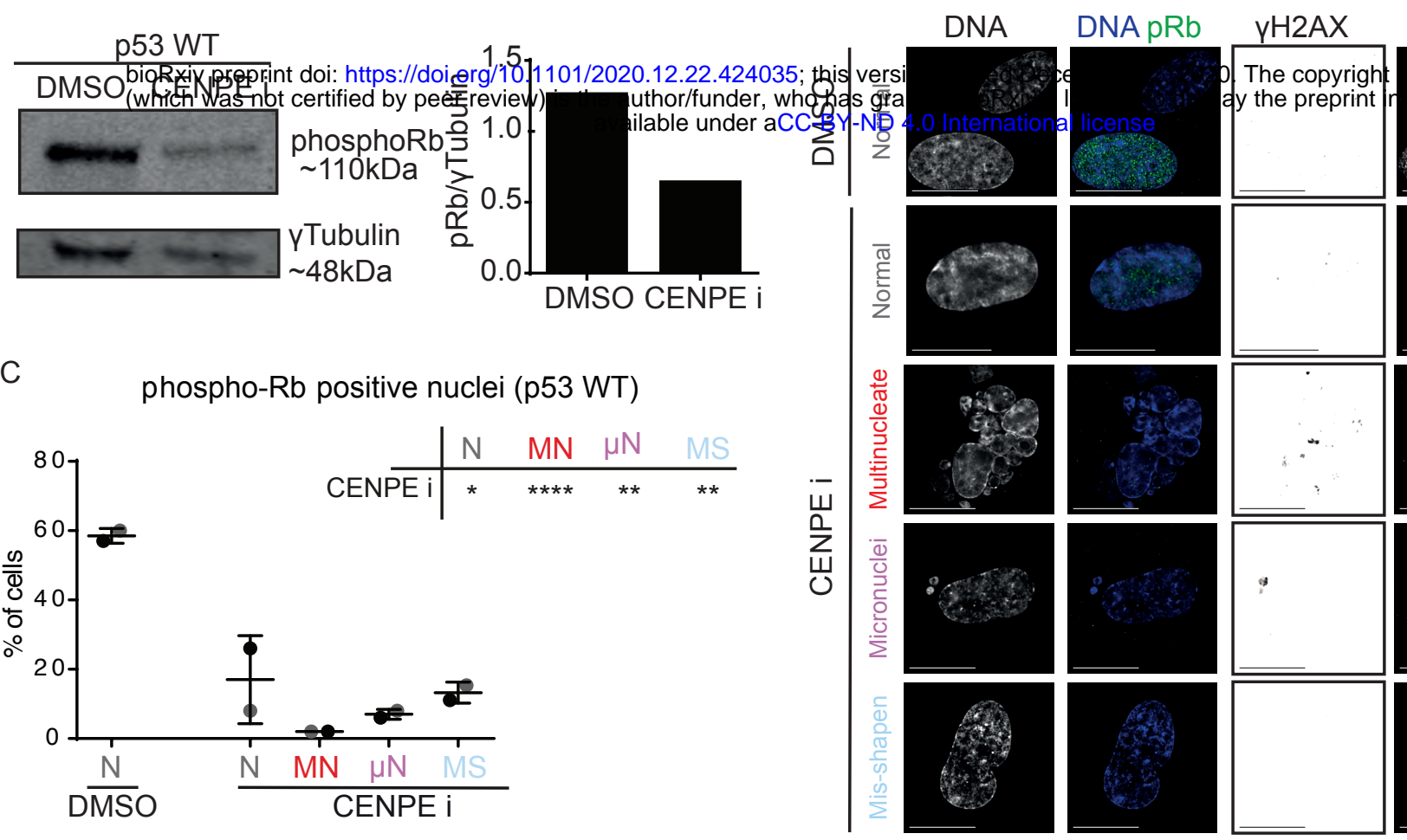

$p R b$

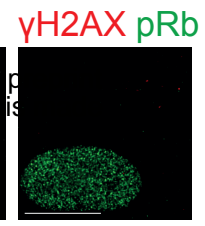

C

phospho-Rb positive nuclei (p53 WT)

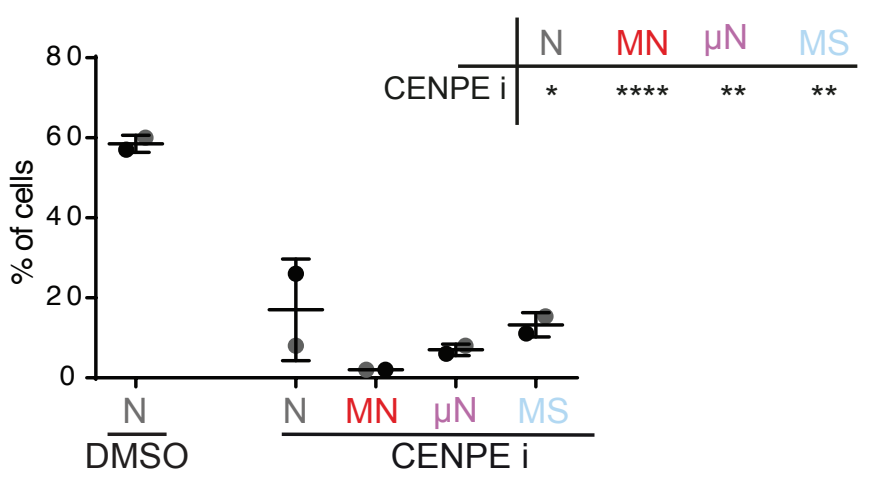

E
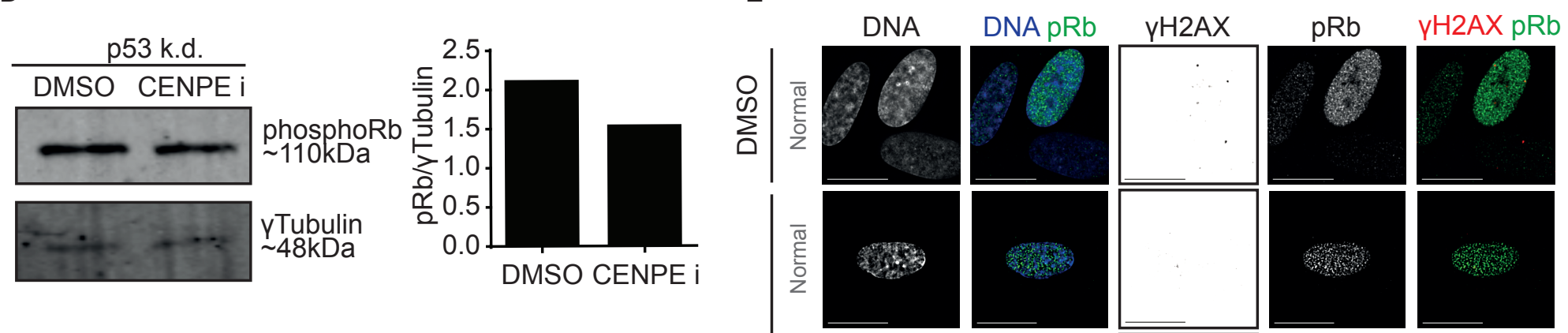

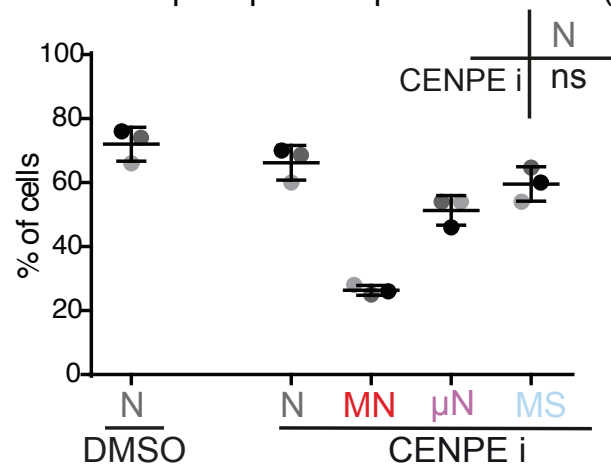

G
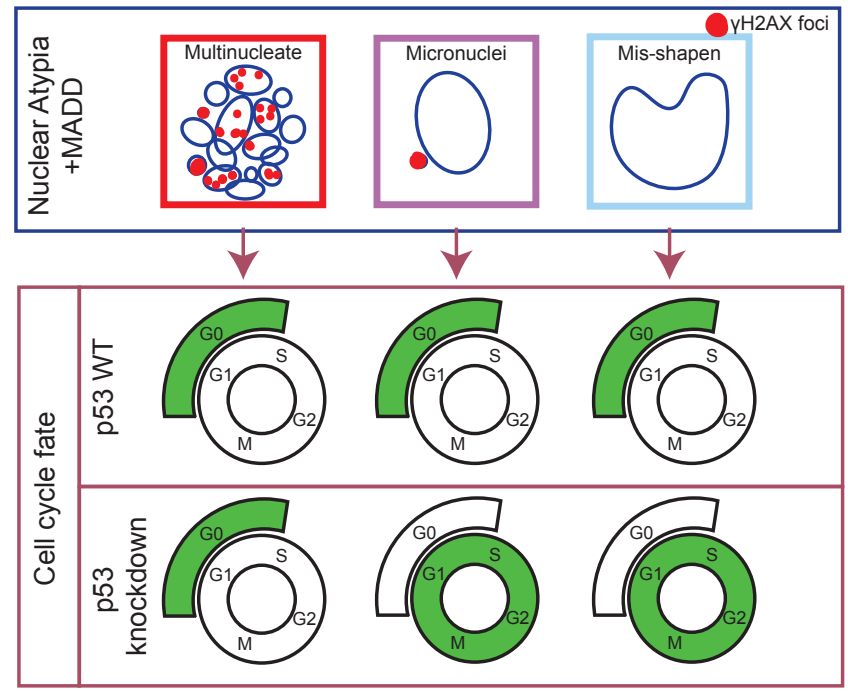

Normal

Multinucleate

Micronuclei

Mis-shapen

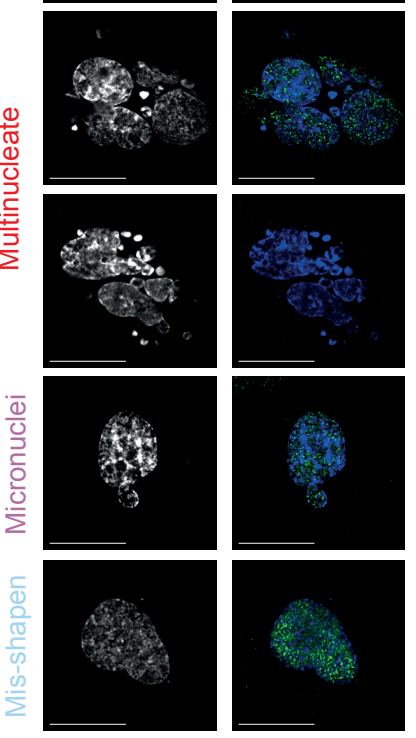
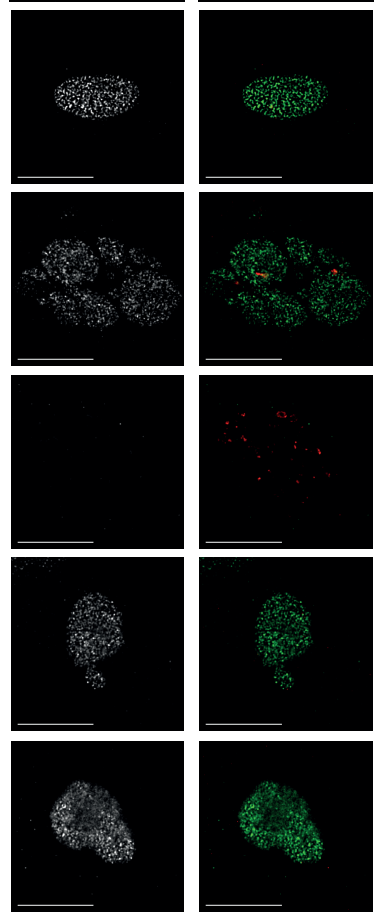